\section{Intersections}

Canadian Journal of Music

Revue canadienne de musique
Intersections CANADIAN JOURAL OF MUSIC
REVUE CANADIENEE DE MUSIOUH

\title{
Something to Sing About: A Preliminary List of Canadian Staged Dramatic Music Since 1867
}

\section{Mary I. Ingraham}

Volume 28, numéro 1, 2007

URI : https://id.erudit.org/iderudit/019291ar

DOI : https://doi.org/10.7202/019291ar

Aller au sommaire du numéro

\section{Éditeur(s)}

Canadian University Music Society / Société de musique des universités canadiennes

\section{ISSN}

1911-0146 (imprimé)

1918-512X (numérique)

Découvrir la revue

Citer cet article

Ingraham, M. I. (2007). Something to Sing About: A Preliminary List of Canadian Staged Dramatic Music Since 1867. Intersections, 28(1), 14-77. https://doi.org/10.7202/019291ar

\section{Résumé de l'article}

Le but de cet article est de présenter - à titre de contribution à un champ d'activités culturelles et de recherche trop souvent négligé - une liste préliminaire d'oeuvres musicales dramatiques composées pour la scène entre 1867 et 2007. Au-delà du simple exercice de recensement d'un répertoire, cet article considère ces oeuvres comme d'importants documents historiques articulant les valeurs contemporaines culturelles, sociales et politiques depuis les 140 dernières années. Suite à l'évaluation des enjeux apparus en déterminant le genre et la citoyenneté musicale des oeuvres, leur ampleur, la nature et l'histoire de celles-ci constituent une solide base permettant la recherche systématique au sein de cet important artefact culturel canadien. Afin de faciliter l'accès à ce corpus substantiel, la liste préliminaire est proposée en trois formats : 1) en ordre alphabétique de noms de compositeurs; 2) en ordre alphabétique des titres de compositions; 3) en ordre chronologique d'achèvement de l'oeuvre. Dans les trois listes, les compositions comportent un identifiant ("PIN » pour Preliminary Ingraham Number) composé du nom du compositeur et de la date d'achèvement de l'oeuvre.
Tous droits réservés (C) Canadian University Music Society / Société de musique des universités canadiennes, 2007
Ce document est protégé par la loi sur le droit d'auteur. L'utilisation des services d'Érudit (y compris la reproduction) est assujettie à sa politique d'utilisation que vous pouvez consulter en ligne.

https://apropos.erudit.org/fr/usagers/politique-dutilisation/ 


\title{
Something to Sing About: A Preliminary List of Canadian Staged Dramatic Music Since 1867
}

\author{
Mary I. Ingraham
}

\begin{abstract}
National literatures are not passive reflections of naturally occurring phenomena, but integral components in the process of national development, consciously constructed pieces of the national culture, and creators of the world in which we live.

The canon is chosen, not born.
\end{abstract}

Sarah Corse

It is the consumer, not the producer, who benefits by culture, the consumer who becomes humanized and liberally educated. There is no reason why a great poet should be a wise and good man, or even a tolerable human being, but there is every reason why his reader should be improved in his humanity as a result of reading him.

Northrop Frye

\section{INTRODUCTION TO THE LIST}

The primary aim of this article is to present a three-fold preliminary list of Canadian staged dramatic music composed between 1867 and 2007 as a contribution to a previously under-cultivated field of Canadian cultural activity and research. A lexical problem arises immediately, however, a problem posed by the following question: what constitutes staged dramatic music in an historically evolving Canadian context? This introduction addresses the question by taking opera as the sine qua non of staged dramatic music, but also by treating it as one component of a broadly encompassing genre entitled staged dramatic music. The genre of opera will serve here from time to time as the measure, the point of reference for this study. The term staged dramatic music, however, will be used with greater frequency so as to indicate the full compass of the genre under question here-compass being the aim of this study.

Assumptions about the aesthetic value of Canadian staged dramatic music, as well as the impact of changes in public and private support for cultural initiatives since Canada's Constitution Act in 1867 (especially those relating to creation, production and audience development) may be at least partially to blame for the lack of such a list to date. At the very least, lack of performance and 
promotion opportunities beyond their premieres has undoubtedly restricted our knowledge of this otherwise rich cultural field. The works listed evidence an evolution in the narrative, form, and function of Canadian staged dramatic music over the past 140 years. Many of these works (both individually and in groups) are important historical documents, articulating contemporaneous cultural, social, and political values. For the purpose of studying the continuum of creativity in these works, and to facilitate access to what is a substantial body of information, this preliminary list is offered in three formats: alphabetically by composer's surname (List 1), alphabetically by the title of the work (List 2), and chronologically by date of completion of the work (List 3). List 1 also indicates the librettist; List 3 includes genre descriptions. Compositions are identified across all formats by a Preliminary Ingraham Number (PIN) comprised of the composer's surname and the date of completion of the work.

Just what constitutes a "Canadian" composer is problematic too, but for the purpose of this study nationality is based on citizenship: a composer who by birth or immigration is Canadian is considered to be a Canadian composer. Further to this, the citizenship of the composer at the time of the creation of the work cited is also a factor in its inclusion. To understand the complexity of this issue, consider that several works in the operatic genre by well known Canadian composers do not appear here, as they were written before the composer immigrated to Canada, such as early works by Theo Goldberg and Bengt Hambreaus. Underlying the issue of nationality are two further assumptions: defining a single musical identity for these works is neither desirable nor possible, and any perceived "Canadian-ness" in any one of these compositions is to be understood as constructed through a complex web of social, political, and cultural influences, this web being initiated and mediated by the individuals and organizations that create, fund, produce, and critique these works.

Music, drama, and staging are the simple foundations of the genre, but it has been described by numerous terms and takes on a wealth of performance forms. Indeed, the term "opera" has been used in Canadian composition to describe forms of staged dramatic and musical works from pantomimes and burlesques to ballads and all manner of musical plays. In selecting works for this list, the primary focus has been on staged repertoire that demonstrates a sustained dramatic and musical continuity ${ }^{1}$, this decision being underscored by a broad definition of the genre of opera. Works identified to date number 379 , and represent at least 184 different composers active in Canada between 1867 and 2007.

Staged dramatic music as a democratic art has existed throughout Canada's history, and a performance in the early post-colonial years was as likely to be a "burlesque in grand opera style" (e.g., Broughall's 1886 Tearful and Tragical Tale of the Tricky Troubadour) as it was to be a grand opera in the European tradition (e.g., Telgmann's 1889 Leo, The Royal Cadet). In summarizing the operatic scenes of two major Canadian centres (Montreal and Toronto) between 1783

1 Although actual performances of all of the works listed cannot be confirmed at this point in the study, it is assumed that the works were staged or that staging was likely. 
and 1980, Dorith Cooper describes the nature of Canadian performance traditions in the genre to include:

[ ... ] opera as "afterpiece" to a play; opera in truncated versions to accommodate the evening's fare normally comprising a play, farce, vaudeville or concert; opera as "mainpiece" to a play, pantomime or ballet; 'costumed' concerts; unstaged opera in isolation with piano accompaniment and with or without costumes and scenic effects; fully staged performances of grand and/or light operas with modest and later complete production accessories. (Cooper 1984, x)

Given this varied representation of the genre, it is expected that additional works may come to light in future that should or could be included in a list of Canadian staged dramatic music. ${ }^{2}$ In fact, genre ambiguity continues to be a significant challenge to compilation of a list of works to the present day, and at present this study and the accompanying list of compositions includes works that might be considered by some as not in an operatic vein, including works presented by organisations or production companies and labeled as "opera" that might be defined more clearly as parodies or compilations, and operas that incorporate folk or popular musical styles.

The twentieth-century musical presents an additional challenge to this study, as this genre also does not sit within clearly-delineated formal boundaries. As Borroff (1984) has noted in her study of American musical theatre, "some musicals are plays and some are not," signifying that those that are considered "plays" (i.e., those that incorporate a sustained dramatic plot) generally include a musical score written by a single composer and "a set of musical numbers conceived as one score with a balanced aesthetic whole." Such works might appropriately be included in this list as examples of original staged dramatic music. Other musicals are conceived without such continuity and are experienced as a series of unrelated "acts" in which the music is "secondary". "In general," Borroff continues, "the musicals that are plays can be described as musically organic, those that are not, as additive" (101). Bernard Williams, contributing to the definition of "Opera" in Grove Music Online, suggests further that connections between opera and other forms of staged dramatic music are both "complex" and "arbitrary", concluding that the issue of distinguishing opera from other forms is more "cultural" than "stylistic". "The present position," he writes, "is that opera is to some extent an evaluative term, used to refer to sung drama which is either 'serious' enough, or traditional enough in form and technique, to be staged in an opera house." ${ }^{3}$ Considerations of "sustained" dramatic and musical plots, the

2 On this point, I would appreciate hearing from anyone who can correct errors or add new information or titles to this database. Contact is preferred by email to: mary.ingraham@ualberta.ca.

3 Elaborating specifically on the relationship of musicals to opera in English-speaking traditions (UK, North America in particular), Williams suggests that:

it is the musical that has played the role in popular taste which, earlier, was played in Vienna by operetta and in Italy by Italian opera itself. To deny that the musical is opera is not simply a stipulation. It originated in part from English $19^{\text {th }}$ century burlesque, and in part from operetta, in particular from the emigration of German composers to the USA, but it has developed in such a way that, at least as things now stand, the styles of dramatic performance and singing 
"seriousness" of intent, use of "traditional" forms and "production" (i.e. validation) by opera companies described by both authors suggests a more inclusive definition of the operatic genre than is generally considered. Preliminary examination indicates that several Canadian works labeled by their creators as 'musicals' might therefore be included in this list of staged dramatic music in future. These works are variously designated as music or musical comedy, farce, play, or theatre. It is understood, however, that closer consideration of the materials of many of these compositions and of the circumstances of their creation and production is required in order to establish their appropriateness for inclusion in this list.

Borroff identifies an approximate chronology for American musical theatre in general that might serve as the basis for historic genre studies of original Canadian staged dramatic music. Her chronology takes into account cross-fertilization of melodrama and pantomime, operetta (of all descriptions), minstrel shows, vaudeville and variety programs, farce, burlesques, and revues. In like manner, Keillor observes, for example, that the Canadian burlesque Broughall 1886 (Tearful and Tragical Tale of the Tricky Troubadour), which is further defined as "in grand opera style", is actually "an adaptation of Verdi's Il Trovatore" (Keillor 2006, 139). Dixon compiled 'Our Boys' in the Riel Rebellion (Dixon 1886) from existing music for a specific community setting, and incorporated a textual narrative intended to comment on local social and political situations. Bengough 1883 (Bunthorne Abroad), a parody of Gilbert and Sullivan, and Broughall 1885 (90th On Active Service), based on then contemporary events involving Louis Riel, are further described as "musical and dramatic burlesques" (ibid.). Three of these-Broughall 1885, Broughall 1886, and Dixon 1886-are the only works contained in this list to be designated as "compiled" from existing music or music by other composers. To the extent that these works present a sustained dramatic plot in a staged setting they are of interest here, although further study of the music may be needed to determine the ultimate importance of the music in the drama.

Minstrel, vaudeville and variety shows, farces, and revues of the early years of Canadian composition-none of these are included in the list at this time, since they typically do not involve the sustained plot line or musical connectivity characteristic of staged dramatic musical works. Quesnel/Ridout 1963 makes an interesting study in this context and in the evolution and acceptance of opera as a genre. The work listed here is a reconstruction by Godfrey Ridout of Joseph Quesnel's 1778 Colas et Colinette, originally designated as a "Comédie en 3 Actes en prose, mêlée d'Ariettes". Quesnel's work did not have an overture and included vaudeville in its epilogue-like conclusion. Written originally as an afterpiece to a play (cf. Cooper, above), Ridout's restoration involved the reconstruction of orchestral parts and the composition of an overture (fourteen vocal 
numbers were extant at the time of the reconstruction ${ }^{4}$ ), in essence both creating and recreating a coherent musical entity in an operatic style.

The majority of works in the preliminary list do in fact have "opera" or "operetta" in their genre description. ${ }^{5}$ Hybrid genre labeling is more prevalent since the middle of the century, due possibly to a shift in focus from the stylistic tradition to a focus on other factors such as the function of the work, its text, its musical or dramatic style, or its intended audience. Original compositions in genres such as pantomime, masque, and pageant, however, appear only occasionally in Canadian repertoire since 1867. Examples of these sub-genres occur nonetheless throughout the period under study: Clappé 1879 (Canada's Welcome), Pannell 1977b, (Circe) and Rolfe 2004b (Orpheus and Eurydice) are masques; Willan 1929a (Indian Christmas [Nativity] Play), Willan 1943 (Brébeuf), Harris 1952 (Portrait of a City), Anhalt 1986 (Winthrop), and Pannell 1990 (The Animals of Limbo) are classified as pageants. Rickard 1993 (Fletcher's Challenge) carries a hybrid genre designation as an "opera pageant". The English pantomime has three exemplars in the list: Burritt 1969 (Once Again ... Pop!), Burritt 1971 (Electric Chair), and Badian 1997 (Scenic Mosaics). ${ }^{6}$

Important sources utilized in the initial stages of this project include Dorith Cooper's 1984 doctoral dissertation on opera in Montreal and Toronto, a printed library catalogue of Canadian opera produced by the Canadian Music Centre (CMC) in 1999, and George Proctor's Canadian Music of the Twentieth Century published in 1980. Cooper provides the most comprehensive information, but along with Proctor it requires updating to include subsequent compositions. The CMC catalogue, also somewhat out of date, contains only their library holdings of unpublished scores composed by their own Associate Composers. Additional sources now widely available in digital formats have also been accessed in order to construct a more complete picture of repertoire across the period. These include materials available in print and online from public institutions such as Library and Archives Canada, further resources from the CMC, print and online publications such as the Encyclopedia of Music in Canada (EMC), Keillor's 2006 Music in Canada, and countless journal and digitized resources such as those prepared by the $\mathrm{CBC}$ and the Canada Council. ${ }^{7}$ Composer's names, work titles, and dates among these sources generally agree, but genre descriptions are not always consistent. Cooper's attention to detail has resulted in the most

4 Keillor $(2006,90)$ describes Quesnel's original work as based on "stock eighteenth-century elements" of character and plot, but which is accompanied by "skillfully written music" that "fills out" the characters.

5 Beyond the pantomimes, burlesques and masques already noted, the most frequent genres in the works included here are: opera, operetta, comic opera, ballad opera, and chamber opera. Including as a group those works that have the word "opera" in their genre description, over fifty different genre labels are used in connection with the works listed.

6 Harriss 1896 (Torquil) is the sole work included in the list with the label "dramatic legend". Keillor $(2006,140)$ refers to this works as "a concert work without action or scenery", which would disqualify it for inclusion here. However, the twenty-three musical numbers employing choral, solo, and orchestral parts suggests otherwise, and the work is included here pending additional research.

7 I am grateful for the assistance of three Graduate Research Assistants at the University of Alberta for help in collecting and organizing this data at various stages of its compilation: (Dr.) Jay Hodgson (2004-2006), Trifon Heaney (2007), and Iain Gillis (2008). 
precise listings, while Proctor lists those he includes mostly under a general genre description of "opera". CMC contains discrepancies between print and online entries and the $E M C$ entries under one heading include works described differently in other parts of the reference. List 3 of this article indicates genre designations found (when there is a reference to genre) in each of five primary sources used to compile the list (Cooper 1984, CMC 1999, CMC online, EMC online, Proctor 1980). The genre of other works has been identified from direct composer sources.

Finally, it must be noted that in the list several works appear with two titles, some with distinct alternate titles and others with a combination of French and English translation that is not always literal. For consistency, double titles follow the format of Grove Music Online, which shows a primary title first and indicates double or split titles within square brackets; where two titles apply, both are cross-referenced in List 2. Titles containing exact translations into either French or English utilize a semicolon to separate them, unless the title itself contains the word 'ou' or 'or'. Works in a series such as those in Schafer's Patria appear in the list as numbered Patria titles followed by a colon and the descriptive title; these works are also cross-referenced in List 2.

\section{Introduction to Canadian Staged Dramatic Music}

The story of original staged dramatic music written and performed on what is now Canadian soil begins long before this nation was born. Its ties to the style and circumstances of repertoire from across the Atlantic are strong in the early years in particular, but long before Canada's Confederation in 1867 modifications were required to make such works suitable for local performers, theatres and audiences. Historiographic work on Canadian music undertaken over the past fifty years has contributed a great deal to our understanding of the living and working conditions of Canadian musicians since colonization (e.g., Kallmann 1960, Proctor 1980, and Keillor 2006). And while the challenges of performing opera in a young colony such as Canada persisted well into the twentieth century, the opportunities for tailoring new works to the sensibilities and interests of audiences in this "new" world were understood almost immediately. The stories of life in Canada proved to be fertile ground for new works in a variety of dramatic genres. ${ }^{8}$

Musical scores for a number of these early staged dramatic musical works are no longer available and many are presumed lost. The challenge of availability and access to primary source material on original Canadian compositions, therefore, has resulted in an approach to Canadian staged dramatic music as a socio-historical phenomenon, an approach that serves ethnomusicologists somewhat better than it does musicologists. Although Canadian music defies canonization in the traditional musicological sense, it is not because the works themselves are not extraordinary (many of them are), but rather, as I have found in my own research in the areas of cultural politics and creation, because con-

8 Consider, for example, the textual and musical resources used in compilations by Broughall and Dixon noted above. 
ditions of production and consumption have separated them from the mainstream. Corse's statement, cited as epigraph to this article, that "the canon is chosen, not born" rings particularly true for the situation of Canadian staged dramatic music (Corse 1997, 9), and the time has come to consider these works on their own terms.

The unique social and political domains affecting Canadian cultural production in general demand consideration of staged dramatic music from the perspectives of both reflective and cultural sociologies that view cultural objects as representative of their environment and that advocate for an informed exploration and analysis of multiple external influences. Research in Canadian culture by scholars outside of the discipline of musicology has much to offer our understanding of composers in Canada and their dramatic works. Important work has been done by Hutcheon (1995, 1990, and others), Mackey (1999), Corse (1997), and Straw (2005) in particular, in areas of multiculturalism, nationalism and identity, and their research suggests that we ought to examine the role of society and politics in the construction of cultural objects such as dramatic musical works. Additionally, cultural sociologists such as Said (1993) and Bourdieu (1993) endorse reflection on the conditions of production and consumption of culture in relation to power structures and the tensions of conflicting interests of multiple communities.

Bourdieu's critical systems of "fields of cultural production" (1993) are particularly appropriate to this study because they are relevant to a wide spectrum of cultural practices (including operatic composition) and may be applied to many aspects of creation and production previously not considered in the creative equation. These include considerations of location (the physical proximity of "players" in the field vis-à-vis each other and in relation to sources of decision-making or "power"), cultural connections (between and among "players"), and elements of taste and governance (in multiple communities-audience, producers, media, funders, etc.) that are generally eschewed by composers as extraneous to the creative process. ${ }^{9}$ Bridging social and cultural theories with cultural practices in this way allows us to consider multiple spheres of influence on staged dramatic music composed in Canada and to advance critical understanding and appreciation of these works in a direction more appropriate to the genre and country of origin.

Developments in the twentieth century have shifted the location of some forms of staged dramatic music, opera in particular, away from the high-culture domains of its largely Western European models towards a middle ground of high- and low-cultures. This may be the result of a combination of factors such as the cross-pollination of musical styles, variations in performance and production forces, changes in audience and musical tastes, and the continuously evolving creative impulses of composers themselves. Nonetheless, the shift is seen to have been gradual (and one might say ongoing) across the period under

9 Northrup Frye's reading of Canadian literature (cited above, and applicable to music creation in Canada) that suggests that the "consumer" is the true benefactor of culture strongly supports the belief underscored here that audiences should in fact be included in such an analysis of cultural objects. 
study. The results of this shift can be classified into three types: works that maintain their "elite" or high-culture status (including grand opera, ballad opera, and much comic opera), works intended as popular repertoires (including music for vaudeville, burlesques, parodies, and musicals), and those created primarily as "educational" works for building audiences (such as radio and television operas, and the more recent trend to short works such as "pocket" operas). Although the compositions included in this preliminary list appear as examples on a continuum of development, each work is unique in regard to some aspect of the production forces required, optimum performance venue, audience demographic targeted, potential or realized sources for funding support, and the nature and mode of critical reception pre- and post-production.

With very few exceptions, Canadian staged dramatic music is not well known even within the sphere of high-culture enthusiasts. Few works have received more than a single performance, and most are accessible only to audiences within a limited geographical area (the area served by the production company responsible for the premiere) ${ }^{10}$ Large-scale works are expensive to produce, to be sure; but ignorance of an entire body of original work has consequences beyond mere economics and impacts the entire cultural field both at home and abroad. The first step in rectifying this situation is to build an awareness of the repertoire of Canadian staged dramatic music, the aim of this article.

\section{Preliminary Results: Presentation of Findings}

The process of compiling this preliminary catalogue of staged dramatic music works has involved a number of challenges, from locating primary source materials to finding definitive descriptions of their contents and details of their creation. Discrepancies in information are surprisingly common in the reference material, with genre definition being the most inconsistent, as noted above. In creating each work's unique PIN, the most problematic element has been finding agreement on a date of completion for the composition. Where completion date cannot be clearly established, the date of the premiere or earliest known performance is used. Where more than one work by a given composer is assumed to have been completed in a single year, these works are listed alphabetically by title, and the designation 'a,' $b$ ', or ' $c$ ' (etc.) is added after the date in the PIN. Compositions for which neither completion nor premiere dates have been found are identified with the abbreviation 'n.d.' followed by a distinguishing noun from the title of the work, e.g. "Charpentier n.d. Ballade" refers to Charpentier's Ballade du fils de l'homme. Very few works in the list are as yet undated. Several entries indicate works completed on a conceptual basis, but these will not be considered truly "complete" for the purposes of cataloguing until they are performed (e.g., Schafer's Patria 7: Asterion).

10 Premiere information for the works in this preliminary list is not included, but is part of a larger study of these works currently underway. The majority of these works are known to have received their premiere in Canada, but some were heard first in the United States, and a few others have never been performed. 
The total number of staged dramatic musical works composed in each decade since Confederation (see fig. 1) illustrates large-scale changes in Canadian staged dramatic music composition and, when considered within the context of extraordinary economic and political events and important federal cultural initiatives such as the centennial celebrations of 1967, suggests parallels between political cultural policy and events in the social sphere. For example, government funding for culture leading up to centennial celebrations in 1967 was followed by a significant rise in support for production companies, audience-building initiatives and opera creation that carried on into the next decade. Similar new funding initiatives for production (e.g., the Canada Council Opera Creation Fund established in 2001) in the early years of this century (albeit following years of decreasing government support) has resulted in a second "wave" of creation that may not yet have reached its peak. Fluctuations in statistics in the early years of staged dramatic music composition may not yet be significant or reliable due to the lack of available resource materials relating to original composition (i.e., more works may yet be "discovered"). Figure 1, then, attempts to document the rise and fall of staged dramatic music composition across decades. Principally, from this figure it can be noted that the vast majority of Canadian works in this genre (77\%) have appeared since $1945 ; 64 \%$ of the total having been completed since 1967. Perhaps surprisingly, it is also evident that through periods of war, economic recession and fiscal restraint since Confederation, staged dramatic music creation may have declined, but it never disappeared entirely.

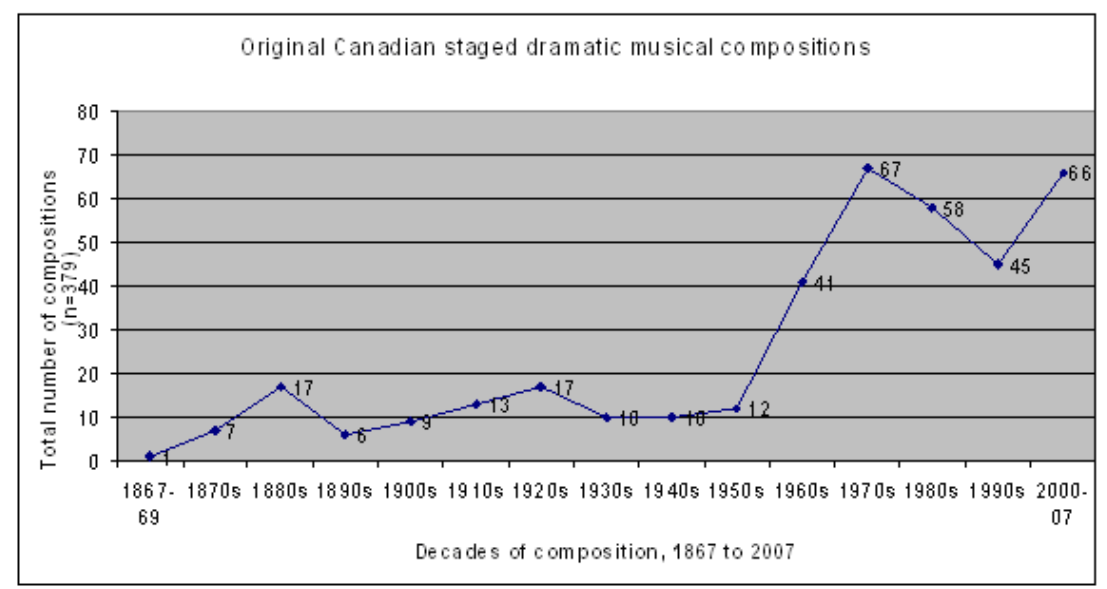

Figure 1. Chart of Original Canadian staged dramatic musical compositions written between 1867 and 2007, showing the total number of works completed in each decade.

An understanding of genre development within specific periods is not evident from Figure 1 alone. Research in these areas will undoubtedly illuminate both small- and large-sale changes to the genre that parallel external events, including changes to the scope and duration of works (the proportionate number of full length staged dramatic musical works to shorter, one-hour or one- 
act compositions, for example), changes resulting from technological pressures (compositions inspired by new media opportunities such as radio and television), and changes resulting from political and economic influences on creation (funding, production, and audience, for example). Broad strokes of change are, however, obvious from the list itself, in the represented narratives in full-scale staged dramatic music compositions, from the early, traditional military opera Leo, the Royal Cadet (Telgmann 1889), through the multi-media, multi-cultural Louis Riel (Somers 1967) in mid-century, to the Asian-Western fusion of musics, instruments and languages in the millennium opera Iron Road (Chan 2000). Still other works demonstrate the scope of staged dramatic music: in terms of duration, for example, the ten-minute chamber opera An English Lesson (Charpentier 1968) lies on one end of a continuum, the dusk-to-dawn rituals performed in Patria 6: RA (Schafer 1983) on the other. The category encompasses structural considerations (Hannan 2002, 120 Songs for the Marquis de Sade), aspects of musical expression (Jackson 2003, Québécité), performing forces (Evangelista 1990, Alice and Friends), and performance venue (Pelletier 1987, Studio variazioni sull'a), all of which continue to challenge generic determination.

Historical periodization of these works is difficult. Pre- and post-Confederation periods make for obvious large-scale chronological breaks, but shorter periodic divisions are less obvious. One might expect to use the establishment of the Canada Council in 1957 as another creative turning point. But as this public institution was not established explicitly to fund the creation of new work, ${ }^{11}$ it is less appealing as a temporal frame for the discussion. More important defining moments in Canadian staged dramatic music might be the start and close of WWI and WWII, and activities leading up to the nation's centennial celebrations in 1967 (which saw increased funding to staged dramatic music production through the National Centennial Commission, Canadian Music Centre, and the Canadian Broadcasting Corporation). The problem of periodization in the years following 1967 is to identify which of the political, fiscal, or cultural events had more influence on the creation of original Canadian work. The details of creation and production undoubtedly hold the secret to understanding how and why certain works appeared on the Canadian scene when they did.

\section{Future Research}

Research that addresses some of the issues raised in the compilation of this preliminary list is at a nascent stage. Opportunities are wide-ranging for research in areas such as the impact that federal cultural policies, public institutional

11 In the section entitled "Part Five: General Observations", Articles 98 (a) and (g) of the Canada Council's First Annual Report the following are stated:

"98 (a) (a) As a general rule the foundation should not initiate projects of its own, and it should not directly commission works or engage artists, but there may be exceptions"

"98 (g) The content of programmes or the choice of plays should not be dictated. Even the performance of Canadian works should not be an express condition of a grant, although the extent to which an organization has presented Canadian artists and works may be considered when an application for a grant or renewal is made." (Canada Council 1958, 25-26). 
practices, and the changing priorities of contemporary society had on the creation of staged dramatic music. The long term impact of genre, location, and the composer's contacts needs study, as does the extent to which the process of creating contemporary staged dramatic music is considered more important than its completion (bearing in mind current practices of workshopping operas at several stages of their development to allow for performer, producer, and audience input).

The findings of research to date indicate a rich field of influences and a variety of cultural objects that are important to an understanding of Canadian historical identity. As social and political priorities have evolved since Confederation, so too have the forms and functions of staged dramatic musical creations, particularly in the choices of texts, styles of musical expression, types of performance and production, and the structural dimensions of the genre itself. Other factors suggest themselves for research, such as who and what gets funded, the subject matter and textual basis of the libretti, types of genres and styles of music as supported by funders, producers, and audience, and the relationship of the many production and reception stages as they affect the creation of the work.

From creation to production and consumption, music is subject to a variety of social and political constraints. In the evolving climate of Canadian cultural production since Confederation, composers have created staged dramatic musical works that reflect Canadian society, that reflect for example the changes in its governance, fiscal, and cultural policies, and the realities of multiculturalism. Works in this genre reflect Canada through the stories of their texts, through structural and technical developments to the genre itself as it adapted to the requirements of particularly Canadian radio and television media (and by adaptation to their fiscal constraints), and through the increased awareness and acceptance of multicultural influences, both textual and musical. Further study into the circumstances of staged dramatic music creation, when combined with analysis of the impact and responses of governments, public institutions, producers, and audiences on these works and on the genre itself, will show that Canadian staged dramatic music both exemplifies the social and political goals of the nation in specific time periods and reflects contemporary practices in musical style and genre. The lists that follow, then, are more than a preliminary exercise in establishing a repertoire. Collectively they constitute a first step in determining a genre by its scope, its nature, and its history, and thereby they lay a foundation for systematic research into this most important Canadian cultural artifact. 


\begin{tabular}{|c|c|c|c|}
\hline Composer & Title & Librettist & Preliminary Ingraham Number \\
\hline Adaskin, Murray & Grant, Warden of the Plains & Bayer, Mary Elizabeth & Adaskin 1967 \\
\hline Adaskin, Murray & Traveling Musicians, The & Page, Grimm & Adaskin 1983 \\
\hline Aldous, John Edward Paul & Golden Catch, A & Aldous, John Edward Paul & Aldous $1902 \mathrm{a}$ \\
\hline Aldous, John Edward Paul & Nancy or, All for Love & Aldous, John Edward Paul & Aldous $1902 \mathrm{~b}$ \\
\hline Aldous, John Edward Paul & Poster Girl, The & Aldous, John Edward Paul & Aldous 1902c \\
\hline Aldous, John Edward Paul & Ptarmigan or, A Canadian Carnival & Mcllwraith, Jean & Aldous 1895 \\
\hline Anhalt, Istavan & Tourangelle, La & Anhalt, Istvan & Anhalt 1975 \\
\hline Anhalt, Istavan & Traces (Tikkum) & Anhalt, Istvan & Anhalt 1996 \\
\hline Anhalt, Istavan & Winthrop & Anhalt, Istvan & Anhalt 1986 \\
\hline Anhalt, Istavan & Millenial Mall (Lady Diotima's Walk) & Anhalt, Istvan & Anhalt 2000 \\
\hline Applebaum, Louis & Erehwon & Moore, Mavor & Applebaum 2000 \\
\hline Archer, Violet & Meal, The & Holt-Wilson, Rowland & Archer 1983 \\
\hline Archer, Violet & Sganarelle & $\begin{array}{l}\text { Archer, Violet; Eliot, Samuel Jr. (based } \\
\text { on Molière) }\end{array}$ & Archer 1973 \\
\hline Arsenault, Raynald & Aristocratic Lover, The & Currie, Ken & Arsenault 1987 \\
\hline Badian, Maya & Scenic Mosaics & Badian, Maya & Badian 1997 \\
\hline Barnes, Milton & Byron, the Wonderful Bandit & Oldfield, Jank; Conway-Marmo, Helen & Barnes 1965 \\
\hline Beckwith, John & Crazy to Kill & Reaney, James (based on Ann Cardwell) & Beckwith 1988 \\
\hline Beckwith, John & Night Blooming Cereus & Reaney, James & Beckwith 1958 \\
\hline Beckwith, John & Shivaree, The & Reaney, James & Beckwith 1978 \\
\hline
\end{tabular}




\begin{tabular}{|c|c|c|c|}
\hline Composer & Title & Librettist & Preliminary Ingraham Number \\
\hline Beckwith, John & Taptoo! & Reaney, James & Beckwith 1993 \\
\hline Behrens, Jack & Lay of Trym, The & Cockburn, C. Keith & Behrens 1968 \\
\hline Bell, Allen Gordon & Turtle Wakes & McNair, Rick & Bell 2001 \\
\hline Bengough, John Wilson & $\begin{array}{l}\text { Bunthorne Abroad or, The Lass That } \\
\text { Loved a Pirate }\end{array}$ & Bengough, John Wilson & Bengough 1883 \\
\hline Benoist, Marius & Légende du vent, La & Brodeur, Léo & Benoist 1974 \\
\hline Benoist, Marius & Onadéga ou la tragédie de lac des bois & Benoist, Marius & Benoist 1977 \\
\hline Benoist, Marius & Rencontre dans l'escalier, La & Benoist, Marius & Benoist n.d. Rencontre \\
\hline Benoist, Marius & Saint-François d'Assise & Benoist, Marius & Benoist n.d. Saint \\
\hline Benoist, Marius & Secret des Amati, Le & Benoist, Marius & Benoist n.d. Secret \\
\hline Betts, Lorne & Riders to the Sea & Synge, John M. & Betts 1955 \\
\hline Betts, Lorne & Woodcarver's Wife, The & Pickthall, Marjorie L.C. & Betts 1960 \\
\hline Bissell, Keith & His Majesty's Pie & Bissell, Keith & Bissell 1966 \\
\hline Bissell, Keith & Miraculous Turnip, The & Bissell, Keith & Bissell 1980 \\
\hline Bissell, Keith & Musical Play, A & Warner, Brigitte & Bissell 1977 \\
\hline Blackburn, Maurice & Mesure de silence, Une; A Silent Measure & $\begin{array}{l}\text { Morisette-Blackburn, Marthe (trans. Guy } \\
\text { Glover) }\end{array}$ & Blackburn 1954 \\
\hline Blackburn, Maurice & Pirouette & Blackburn, Marthe & Blackburn 1960 \\
\hline Bottenberg, Wolfgang & Inook & Beissel, Henry & Bottenberg 1986 \\
\hline Brégent, Michel-Georges & Réalitillusion & Brégent, Michel-Georges & Brégent 1988 \\
\hline
\end{tabular}




\begin{tabular}{|c|c|c|c|}
\hline Composer & Title & Librettist & Preliminary Ingraham Number \\
\hline Broughall, George (compiler) & $\begin{array}{l}\text { 90th on Active Service, The; or, } \\
\text { Campaigning in the North West }\end{array}$ & Broughall, George & Broughall 1885 \\
\hline Broughall, George (compiler) & $\begin{array}{l}\text { Tearful and Tragical Tale of the Tricky } \\
\text { Troubadour, The; or, The Truant Tracked }\end{array}$ & Broughall, George & Broughall 1886 \\
\hline Buck, Sadie & Bones & Buck, Sadie & Buck 2001 \\
\hline Buczynski, Walter & From The [Buczynski] Book of the Living & Buczynski, Walter & Buczynski 1972 \\
\hline Buczynski, Walter & Naked at the Opera & Hendry, Tom & Buczynski 1978 \\
\hline Burritt, Lloyd & Altar of the Sun & Burritt, Lloyd & Burritt 1983 \\
\hline Burritt, Lloyd & Electric Chair & Burritt, Lloyd & Burritt 1971 \\
\hline Burritt, Lloyd & Once Again . . . Pop! & Burritt, Lloyd & Burritt 1969 \\
\hline Burritt, Lloyd & Pilgrim [The Dream Healer] & $\begin{array}{l}\text { Findley, Timothy; Christopher Allen; [rev. } \\
\text { by Don Mowatt] }\end{array}$ & Burritt 2003 \\
\hline Burry, Dean & Brothers Grimm, The & Burry, Dean & Burry 2001 \\
\hline Burry, Dean & Hobbit, The & Burry, Dean (based on J.R.R. Tolkein) & Burry 2004 \\
\hline Burry, Dean & Isis and the Seven Scorpions & Burry, Dean & Burry 2006a \\
\hline Burry, Dean & Shanawdithit & Burry, Dean & Burry 1996 \\
\hline Burry, Dean & Unto the Earth: Vignettes of a War & Burry, Dean & Burry 1993 \\
\hline Burry, Dean & Vinland Traveler, The & Burry, Dean & Burry 2006b \\
\hline Butterfield, Christopher & Zurich 1916 & Mays, John Bentley & Butterfield 1998 \\
\hline Cabena, Barrie & Selfish Giant, The & Cabena, Barrie (based on Oscar Wilde) & Cabena 1970 \\
\hline Chan, Ka Nin & Ice Time & Brownell, Mark & Chan 2004 \\
\hline
\end{tabular}




\begin{tabular}{|c|c|c|c|}
\hline Composer & Title & Librettist & Preliminary Ingraham Number \\
\hline Chan, Ka Nin & Iron Road & Brownell, Mark & Chan 2000 \\
\hline Charpentier, Gabriel & Ballade du fils de l'homme & Charpentier, Gabriel & Charpentier n.d. Ballade \\
\hline Charpentier, Gabriel & Bourgeois gentilhomme, Le & after Molière & Charpentier 1964 \\
\hline Charpentier, Gabriel & $\begin{array}{l}\text { Clara et les philosophes; Clara and the } \\
\text { Philosophers }\end{array}$ & Char, Benoit & Charpentier 1976 \\
\hline Charpentier, Gabriel & Clara on the Bridge & Bosco, Monique & Charpentier n.d. Clara Bridge \\
\hline Charpentier, Gabriel & Clara, la nuit & Blais, Marie-Claire; Charpentier, Gabriel & Charpentier n.d. Clara Nuit \\
\hline Charpentier, Gabriel & Clara, un masque & Charpentier, Gabriel & Charpentier n.d. Clara masque \\
\hline Charpentier, Gabriel & Clarabelle-Clarimage & Char, Benoit (trans. N. Cote) & Charpentier 1979 \\
\hline Charpentier, Gabriel & Claracello ou Répertoire & Charpentier, Gabriel & Charpentier 1985 \\
\hline Charpentier, Gabriel & Clara-Committee & Tremblay, Michel & Charpentier n.d. Clara Committee \\
\hline Charpentier, Gabriel & English Lesson, An; or, Clara-Teacher & Charpentier, Gabriel & Charpentier 1968 \\
\hline Charpentier, Gabriel & Klondyke & Languirand, Jacques & Charpentier 1965 \\
\hline Charpentier, Gabriel & Orphée I & $\begin{array}{l}\text { Charpentier, Gabriel (trans. Michael } \\
\text { Bawtree) }\end{array}$ & Charpentier 1969 \\
\hline Charpentier, Gabriel & Orphée II & $\begin{array}{l}\text { Charpentier, Gabriel (trans. Michael } \\
\text { Bawtree) }\end{array}$ & Charpentier 1972a \\
\hline Charpentier, Gabriel & $\begin{array}{l}\text { Tea Symphony, The; or, The Perils of } \\
\text { Clara }\end{array}$ & Charpentier, Gabriel & Charpentier $1972 b$ \\
\hline Clappé, Arthur A. & Canada's Welcome: A Masque & Dixon, Frederick Augustus & Clappé 1879 \\
\hline Coulthard, Jean & Return of the Native, The & $\begin{array}{l}\text { Baxter, Edna; Coulthard, Jean (based on } \\
\text { Thomas Hardy) }\end{array}$ & Coulthard 1956 \\
\hline
\end{tabular}


List 1: (cont'd)

\begin{tabular}{|c|c|c|c|}
\hline Composer & Title & Librettist & Preliminary Ingraham Number \\
\hline Crawley, Clifford & Angel Square & Irwin, Janet & Crawley 1996 \\
\hline Crawley, Clifford & Barnardo Boy & Helwig, David & Crawley 1980 \\
\hline Crawley, Clifford & Pied Piper, The & Helwig, David & Crawley 1989 \\
\hline Crawley, Clifford & Slaughter of the Innocents, The & Wright, Robert (Based on Martin Luther) & Crawley 1974 \\
\hline Current, Brian & Airline Icarus & Piatogorsky, Anton & Current 2006a \\
\hline Current, Brian & Inventory & Current, Brian & Current 2006b \\
\hline Daniel, Omar & Lisa & Poch-Goldin, Alex & Daniel 2002 \\
\hline Daniel, Omar & Shadow, The & Poch-Goldin, Alex & Daniel 2007 \\
\hline Daniel, Omar & Via Cruxus & Daniel, Omar (Basedon martin Luther) & Daniel 2004 \\
\hline Danielson, Janet & Mariken of Nimmegen & $\begin{array}{l}\text { Danielson, Janet (based on an anonym- } \\
\text { ous 15th century play) }\end{array}$ & Danielson 2003 \\
\hline Davies, Victor & Beowulf & Wylie, B. J. & Davies 1974 \\
\hline Davies, Victor & Let Us Pay Tribute to Lord Gordon & Weatherhead, G & Davies 1968 \\
\hline Delaney, Wiliam & Prince Tommy & Delaney, Wiliam & Delaney 1898 \\
\hline Deschênes, Marcelle & OPéRAaaAH! & Deschênes, Marcelle & Deschênes 1983 \\
\hline Désilets, Richard & Zaâ le loup & $\begin{array}{l}\text { Désilets, Richard (based on Benoit } \\
\text { Robitaille) }\end{array}$ & Désilets 1997 \\
\hline Désilets, Richard & Zaria & Gosselin, Kim Élaine & Désilets 1990 \\
\hline Désilets, Richard & Zoé & Gosselin, Kim Élaine & Désilets 1988 \\
\hline Detweiler, Alan & Beware of the Wolf & Reynolds, Alfred & Detweiler 1964 \\
\hline
\end{tabular}




\begin{tabular}{|c|c|c|c|}
\hline Composer & Title & Librettist & Preliminary Ingraham Number \\
\hline Dixon, L. (compiler) & $\begin{array}{l}\text { Halifax to the Saskatchewan: 'Our Boys' } \\
\text { in the Riel Rebellion }\end{array}$ & $\begin{array}{l}\text { Dixon, L (based on songs by R. } \\
\text { Blackmore, C. Munro, S.H. Romans) }\end{array}$ & Dixon 1886 \\
\hline Dolin, Samuel & Drakkar & $\begin{array}{l}\text { Dolin, Samuel; Dolin, Leslie (based on } \\
\text { the saga of Erik the Red) }\end{array}$ & Dolin 1972 \\
\hline Donison, Christopher & Eyes on the Mountain & Hodgins, Jack & Donison 2001 \\
\hline Donison, Christopher & Island & MacLeod, Alistair & Donison 2006 \\
\hline Doolittle, Quenten & Boiler Room Suite & Deverell, Rex & Doolittle 1988 \\
\hline Doolittle, Quenten & Charlie the Chicken & $\begin{array}{l}\text { Doolittle, Quenten (based on Jonathan } \\
\text { Levy) }\end{array}$ & Doolittle 1975 \\
\hline Doolittle, Quenten & Second Shepherds' Play, The & Wylie, Betty Jane & Doolittle 1980 \\
\hline Doolittle, Quenten & Silver City & Truss, Jan & Doolittle 1984 \\
\hline Doyle, Joseph Nevin & Cingalee & unknown & Doyle n.d. Cingalee \\
\hline Doyle, Joseph Nevin & Enchanted Garden, The & unknown & Doyle 1916 \\
\hline Doyle, Joseph Nevin & Golden Age, The & Doyle, Joseph Nevin & Doyle 1915 \\
\hline Duncan, Laurie & Belle et la Bête, La & $\begin{array}{l}\text { Duncan, Laurie (based on Jeanne-Marie } \\
\text { LePrince de Beaumont) }\end{array}$ & Duncan 2004 \\
\hline Duncan, Laurie & Fall of the House of Usher & Duncan, Laurie (based on Poe) & Duncan 2001 \\
\hline Eggleston, Ann & Woodcarver's Wife, The & Eggleston, Anne & Eggleston 1961 \\
\hline Estacio, John & Filumena & Murrell, John & Estacio 2003 \\
\hline Estacio, John & Frobisher & Murrell, John & Estacio 2006 \\
\hline Evangelista, José & Alice and Friends & Carol, Lewis & Evangelista 1990 \\
\hline
\end{tabular}


List 1: (cont'd)

\begin{tabular}{|c|c|c|c|}
\hline Composer & Title & Librettist & Preliminary Ingraham Number \\
\hline Evangelista, José & $\begin{array}{l}\text { Exercices de conversation et de diction } \\
\text { françaises pour étudiants américains }\end{array}$ & Ionesco, Eugene & Evangelista 1992 \\
\hline Evangelista, José & Manuscrit trouvé à Saragosse & Nous, Alex & Evangelista 2001 \\
\hline Faith, Percy & Gandy Dance, The & Faith, Percy & Faith 1943 \\
\hline Farrell, Dennis & Birthday of the Infanta, The & Farrell, Dennis & Farrell 1979 \\
\hline Ferguson, Sean & Two Graces, The & Diamond, Alexis & Ferguson 2004 \\
\hline Ford, Clifford & Hypnos & Peglar, Kenneth & Ford 1972 \\
\hline Freedman, Harry & Abracadabra & Moore, Mavor & Freedman 1979 \\
\hline Freedman, Harry & Silents! & Moore, Mavor & Freedman 1978 \\
\hline Frenette, Claude & Étrivières, Les & Derosiers, Nichole & Frenette 1983 \\
\hline Fuller, William Henry & $\begin{array}{l}\text { HMS Parliament or, The Lady Who Loved } \\
\text { a Government Clerk }\end{array}$ & Fuller, William Henry & Fuller 1879 \\
\hline Gagné, Marc & Évangéline et Gabriel & Gagné, Marc (inspired by Longfellow) & Gagné 1994 \\
\hline Gagné, Marc & Menaud (Master Logdriver) & Gagné, Marc (after Felix-Antoine Savard) & Gagné 1986 \\
\hline Gagné, Marc & Verdi, Les & Gagné, Marc & Gagné 2001 \\
\hline Gagnon, André & Nelligan & Tremblay, Michel & Gagnon 1990 \\
\hline Geddes-Harvey, Roberta & $\begin{array}{l}\text { Terre Bonne, La; or, The Land of the } \\
\text { Maple Leaf }\end{array}$ & Klugh, A. & Geddes-Harvey 1903 \\
\hline George, Graham & Evangeline & $\begin{array}{l}\text { Roddick, Paul; Warren, Don; (rev. Robert } \\
\text { George) }\end{array}$ & George 1948 \\
\hline George, Graham & King for Corsica, $A$ & George, Graham & George 1975 \\
\hline George, Graham & Revolt in Upper Canada & George, Graham & George 1987 \\
\hline
\end{tabular}




\begin{tabular}{|c|c|c|c|}
\hline Composer & Title & Librettist & Preliminary Ingraham Number \\
\hline George, Graham & Way Out & George, Graham & George 1960 \\
\hline Gibbon, John Murray (compiler) & Jolly Beggars, The & based on songs by Robert Burns & Gibbon 1928 \\
\hline Gilliland, Alan & Hannaraptor & Brandt, Val; Gilliland, Alan & Gilliland 2007 \\
\hline Gingras, Rolland-Georges & Appel du missionnaire, $L^{\prime}$ & Corriveau, J.-Eugène & Gingras 1925 \\
\hline Goldberg, Theo & Concrete Rose, The & Goldberg, Theo & Goldberg 1970 \\
\hline Goldberg, Theo & Galatea elettronica & $\begin{array}{l}\text { Goldberg, Theo (includes writings of } \\
\text { Leonardo da Vinci) }\end{array}$ & Goldberg 1969 \\
\hline Gotham, Nic & Nigredo Hotel & MacDonald, Ann-Marie & Gotham 1992 \\
\hline Gougeon, Denis & Expensive Embarrassment, An & $\begin{array}{l}\text { Anderson, Timothy J (based on Anton } \\
\text { Chekhov) }\end{array}$ & Gougeon 1989 \\
\hline Greer, John & Star-Child, The & Dicken, Ned & Greer 2000 \\
\hline Hambraeus, Bengt & Ouï-dire, $L^{\prime}$ & Hambreaus, Bengt & Hambraeus 1986 \\
\hline Hambraeus, Bengt & Sagan (The Legend) & Runsten, Lars & Hambraeus 1979 \\
\hline Hannah, Ronald & Madamoiselle Fifi & $\begin{array}{l}\text { Holt-Wilson, Rowland (based on Guy de } \\
\text { Maupassant) }\end{array}$ & Hannah 1983 \\
\hline Hannan, Peter & 120 Songs for the Marquis de Sade & Hinton, Peter & Hannan 2002 \\
\hline Hannan, Peter & Gang, The & Cone, Tom & Hannan 1999 \\
\hline Harris, Neil & Portrait of a City & Harris, Neil & Harris 1952 \\
\hline Harrison, Susie & Pipandor & Dixon, Frederick Augustus & Harrison 1884 \\
\hline Harriss, Charles Albert Edwin & Admiral, The & Harriss, Charles Albert & Harriss 1902 \\
\hline Harriss, Charles Albert Edwin & Torquil & Oxenford, Edward & Harriss 1896 \\
\hline
\end{tabular}


List 1: (cont'd)

\begin{tabular}{|c|c|c|c|}
\hline Composer & Title & Librettist & Preliminary Ingraham Number \\
\hline Hatch, Peter & Asks Alice & Stein, Gertrude & Hatch 1998 \\
\hline Hatzis, Christos & Constantinople & $\begin{array}{l}\text { Hatzis, Christos (based on Ancient Greek } \\
\text { texts) }\end{array}$ & Hatzis 2004 \\
\hline Healey, Derek & Seabird Island & Newton, Norman (after a Tsimshian story) & Healey 1977 \\
\hline Henderson, Ruth Watson & Clear Sky \& Thunder & Peterson, Leonard & Henderson 1983 \\
\hline Hétu, Jacques & Prix, Le & Beauchemin, Luc & Hétu 1992 \\
\hline Higgin, Clifford & Queen of Romance, The & Higgin, Clifford & Higgin 1946 \\
\hline Higgin, Clifford & Rumplestilskin & Higgin, Clifford (based on Tom Tit Tot) & Higgin 1945 \\
\hline Hodkinson, Sydney & Saint Carmen of the Main & Tremblay, Michel (adapted by Lee Devin) & Hodkinson 1988 \\
\hline Holman, Derek & Doctor Canon's Cure & Davies, Robertson & Holman 1982 \\
\hline Hone, Jules & Grandee, The & Forsyth, Captain & Hone 1899 \\
\hline Horner, Ralph & Amy Rosbart & Horner, Ralph & Horner n.d Amy \\
\hline Horner, Ralph & Bells of Barcelona, The & Horner, Ralph & Horner 1911 \\
\hline Hui, Melissa & Cellar Door, The & Sy, Jovanni & Hui 2002 \\
\hline Jackson, D.D. & Québécité & Clarke, George Elliott & Jackson 2003 \\
\hline Jackson, D.D. & Trudeau: Long March/Shining Path & Clarke, George Elliott & Jackson 2007 \\
\hline $\begin{array}{l}\text { Jaeger, David; in collaboration with Lake, } \\
\text { Larry and Montgomery, James }\end{array}$ & Nightbloom & $\begin{array}{l}\text { Mulcahy, Sean; Bridgman, Billie (based } \\
\text { on James Joyce) }\end{array}$ & Jaeger, Lake, Montgomery 1984 \\
\hline Jones, Kelsey & Sam Slick & Jones, Rosabelle & Jones 1965 \\
\hline Jones, Trevor & Scottish Landing & Wetmore, Donald & Jones 1967 \\
\hline
\end{tabular}




\begin{tabular}{|c|c|c|c|}
\hline Composer & Title & Librettist & Preliminary Ingraham Number \\
\hline Jones, Trevor Morgan & Broken Ring, The & Wetmore, Donald & Jones 1953 \\
\hline Keane, David & Carmina Tenebrarum & Keane, David & Keane 1983 \\
\hline Keane, David & $\begin{array}{l}\text { Devil's Constructs, The; or, The Eighth } \\
\text { Day }\end{array}$ & Fanstone, David & Keane 1978 \\
\hline Kenins, Talivaldis & Lagalai-Legend of the Stone & Fogels, Uldis (after E. Pauline Johnson) & Kenins 1970 \\
\hline Kerrison, John Davenport & Curfew, The & Kerrison, John Davenport & Kerrison $1879 a$ \\
\hline Kerrison, John Davenport & Last of the Aztecs, The & Kerrison, John Davenport & Kerrison 1914 \\
\hline Kerrison, John Davenport & Maid of the Mill, The & Kerrison, John Davenport & Kerrison $1879 b$ \\
\hline Kerrison, John Davenport & Oreads & Kerrison, John Davenport & Kerrison 1870 \\
\hline Klein, Lothar & Orpheus & Klein, Lothar & Klein 1976 \\
\hline Komorous, Rudolf & Lady Blancarosa & Komorous, Rudolf & Komorous 1966 \\
\hline Komorous, Rudolf & No No Miya & $\begin{array}{l}\text { Motokyio, Deami (adapted by Rudolf } \\
\text { Komorous) }\end{array}$ & Komorous 1988 \\
\hline Koprowski, Peter Paul & Dulcitius: Demise of Innocence & $\begin{array}{l}\text { Anderson, Rodney J (based on medieval } \\
\text { miracle play) }\end{array}$ & Koprowski 1989 \\
\hline Kulesha, Gary & Last Duel, The & Albano, Michael & Kulesha 1999 \\
\hline Kulesha, Gary & Red Emma & Bolt, Carol & Kulesha 1993 \\
\hline Kunz, Alfred & Ceyx and Alcyone & Cummings, Laurence & Kunz 1979 \\
\hline Kunz, Alfred & Damask Drum, The & Hecken & Kunz 1962a \\
\hline Kunz, Alfred & Let's Make a Carol & Reaney, James & Kunz 1965a \\
\hline Kunz, Alfred & Three Figures in a Crystal Ball & unknown & Kunz 1962b \\
\hline
\end{tabular}


List 1: (cont'd)

\begin{tabular}{|c|c|c|c|}
\hline Composer & Title & Librettist & Preliminary Ingraham Number \\
\hline Kunz, Alfred & Watchful Gods, The & Kunz, Alfred & Kunz 1965b \\
\hline Labelle, Jean-Baptiste & $\begin{array}{l}\text { Conversion d'un pêcheur de la Nouvelle- } \\
\text { Écosse, La }\end{array}$ & Labelle, Elzéar; Labelle, Jean-Baptiste & Labelle 1884 \\
\hline Lake, Larry & Tis Pity & Lake, Larry & Lake 1990 \\
\hline Laliberté, Alfred & Sœur Béatrice & Maeterlinck, Maurice & Laliberté 1920 \\
\hline Lamarche, André & Mira & unknown & Lamarche 1981 \\
\hline Lapierre, Eugène & Père des Amours, Le & $\begin{array}{l}\text { Lapierre, Eugène (based on the life of } \\
\text { Joseph Quesnel) }\end{array}$ & Lapierre 1942 \\
\hline Lapierre, Eugène & Vagabond de la gloire, Le & $\begin{array}{l}\text { Plamondon, Aimé (based on the life of } \\
\text { Calixa Lavallée) }\end{array}$ & Lapierre 1947 \\
\hline Lavallée, Calixa & Jugement de Salomon, Le & unknown & Lavallée 1886 \\
\hline Lavallée, Calixa & Loulou & Thiers, Arnold de & Lavallée 1872 \\
\hline Lavallée, Calixa & Veuve, La; The Widow & Nelson, Frank $\mathrm{H}$. & Lavallée 1880 \\
\hline Lavallée-Smith, Alphonse & Gisèle & Lavallée-Smith, Alphonse & Lavallée-Smith 1911 \\
\hline Lavigueur, Célestin & Enfants du manoir, Les & Lavigueur, Celestin & Lavigueur 1881a \\
\hline Lavigueur, Célestin & Fiancée des bois, La & Lemay, Pamphile & Lavigueur 1881b \\
\hline Lavigueur, Célestin & Mariage improvisé, Un & Lavigueur, Celestin & Lavigueur $1881 \mathrm{c}$ \\
\hline Leonard, John & Girl of Bandolier, The & Leonard, John & Leonard 1952 \\
\hline Leonard, John & Maids of Hamelin & Leonard, John & Leonard 1943 \\
\hline Létourneau, Omer & Coup de Soleil & Rousseau, Alfred & Létourneau 1930 \\
\hline Létourneau, Omer & Mam'zell Bebe & Corriveau, J.-Eugene; Plamondon, Aimé & Létourneau 1933 \\
\hline
\end{tabular}




\begin{tabular}{|c|c|c|c|}
\hline Composer & Title & Librettist & Preliminary Ingraham Number \\
\hline Létourneau, Omer & Vive la Canadienne & Corriveau, J.-Eugene; Plamondon, Aimé & Létourneau 1924 \\
\hline Levin, Gregory & Ghost Dance & Moore, Mavor & Levin 1985 \\
\hline Levin, Gregory & Rebel and Empire & Levin, Dan; Levin, Gregory & Levin 1978 \\
\hline Louie, Alexina & $\begin{array}{l}\text { Burnt Toast: } 8 \text { mini comic operas about } \\
\text { love }\end{array}$ & Redican, Dan & Louie 2006 \\
\hline Louie, Alexina & Scarlet Princess, The & Hwang, David Henry & Louie 2002 \\
\hline Louie, Alexina & Toothpaste & Redican, Dan & Louie 2001 \\
\hline Lucas, Clarence & Anne Hathaway & Lewis, Monk & Lucas 1887 \\
\hline Lucas, Clarence & Arabia & Lucas, Clarence & Lucas 1886 \\
\hline Lucas, Clarence & Money Spider, The & Lucas, Clarence & Lucas 1897 \\
\hline Lucas, Clarence & Peggy Machree & Lucas, Clarence & Lucas 1904 \\
\hline Lucas, Clarence & $\begin{array}{l}\text { Puffe \& Co. or, Hamlet, Prince of Dry } \\
\text { Goods }\end{array}$ & Bengough, John Wilson & Lucas 1889 \\
\hline Lucas, Clarence & Semiramis & Wells, Sidthorpe & Lucas 1884 \\
\hline Luedeke, Raymond & $\begin{array}{l}\text { Oscar Excerpts: The Picture of Dorian } \\
\text { Gray }\end{array}$ & Luedeke, Raymond & Luedeke 1996 \\
\hline Luengen, Ramona & Naomi's Road & Hodges, Ann (based on Joy Kogawa) & Luengen 2005 \\
\hline MacDonald, Andrew P. & Unbelievable Glory of Mr. Sharp, The & Koebke, Ken & MacDonald 1989 \\
\hline MacIntyre, David K. & Architect, The & Cone, Tom & MacIntyre 1994 \\
\hline MacIntyre, David K. & Humulus the Mute & $\begin{array}{l}\text { Anouilh, Jean; Aurench, Jean (trans. } \\
\text { Michael Benedict) }\end{array}$ & MacIntyre 1977 \\
\hline MacIntyre, David K. & Refrains & Perrault, Jean-Pierre & MacIntyre 1981 \\
\hline
\end{tabular}


List 1: (cont'd)

\begin{tabular}{|c|c|c|c|}
\hline Composer & Title & Librettist & Preliminary Ingraham Number \\
\hline Maclagan & Queen's Shilling, The & Maclagan & Maclagan 1880 \\
\hline MacMillan, Ernest & Prince Charming & Middleton, J.E. & MacMillan 1931 \\
\hline Major, Douglas & Loyalists, The & Collins, Patricia & Major 1967 \\
\hline Mann, Leslie & Donkey's Tale, The & Mann, Leslie & Mann 1971 \\
\hline Manning, Edward B. & Rip Van Winkle & Manning, Edward & Manning 1919 \\
\hline Mather, Bruce & Princesse blanche, La & Tremblay, Renald (after R.M. Rilke) & Mather 1993 \\
\hline McIntyre, Paul & Death of a Hired Man, The & Frost, Robert & McIntyre 1966a \\
\hline McIntyre, Paul & Thirteen Hands & Shields, Carol & McIntyre 2000 \\
\hline McIntyre, Paul & This Is Not True & Schevill, James & McIntyre 1966b \\
\hline Mcley, David & Salmon Dance & Roth, Evelyn & Mcley 1978, rev 1996 \\
\hline McPeek, Ben & Bargain, The & $\begin{array}{l}\text { McPeek, Benjamin D. (based on the } \\
\text { Faust legend) }\end{array}$ & McPeek 1963 \\
\hline Miller, Michael R. & Around Alline & Miller, Michael & Miller 1989 \\
\hline Mills, Frederick W. & Maire of St. Brieux, The & Dixon, Frederick Augustus & Mills 1875 \\
\hline Miro, Henri & Lolita & Robi, Armand & Miro 1922 \\
\hline Miro, Henri & Million Dollar Girl, A; Le petit Milliardaire & Robi, Armand & Miro 1915 \\
\hline Miro, Henri & Roman de Suzon, Le & Letondal, Rad; Letondal, Henri & Miro 1914 \\
\hline Morgan, Pamela & Nobleman's Wedding, The & Morgan, Pamela & Morgan 1982 \\
\hline Morin-Labrecque, Albertine & Francine & Lamontagne-Beauregard, Blanche & Morin-Labrecque 1930 \\
\hline Nelson, Mr. & Hay Makers, The & unknown & Nelson 1869 \\
\hline
\end{tabular}




\begin{tabular}{|c|c|c|c|}
\hline Composer & Title & Librettist & Preliminary Ingraham Number \\
\hline O'Brien, Oscar & Noce canadienne-française, Une & Bourgeois, Alberic & O'Brien 1930a \\
\hline O'Brien, Oscar & Philippino & Valois, Gaetan & O'Brien 1933 \\
\hline O'Brien, Oscar & $\begin{array}{l}\text { Scènes des voyageurs [Forestiers et } \\
\text { voyageurs] }\end{array}$ & Montigny, Louvigny de & O’Brien 1928 \\
\hline O'Brien, Oscar & Visions canadiennes: Á Saint Malo & O'Brien, Oscar & O'Brien 1930b \\
\hline Ogborn, David & Opera on the Rocks & $\begin{array}{l}\text { Brodie, Leanna; Carley, Dave; } \\
\text { Codrington, Lisa; Dalby, Krista }\end{array}$ & Ogborn 2007 \\
\hline Oliver, John & Guacamayo's Old Song and Dance & Cameron, Melissa & Oliver 1991 \\
\hline Palmer, Juliet Kiri & Over the Japanese Sea & Salverson, Julie & Palmer 2003 \\
\hline Palmer, Juliet Kiri & Shelter & Salverson, Julie & Palmer 2007 \\
\hline Pannell, Raymond & Aberfan & Pannell, Beverly & Pannell 1977a \\
\hline Pannell, Raymond & Animals of Limbo, The & Pannell, Raymond & Pannell 1990 \\
\hline Pannell, Raymond & Aria da Capo & after a play by Edna St Vincent Millay & Pannell 1963 \\
\hline Pannell, Raymond & Circe & Atwood, Margaret & Pannell 1977b \\
\hline Pannell, Raymond & Exiles & Pannell, Beverly & Pannell 1973 \\
\hline Pannell, Raymond & Go & Pannell, Raymond & Pannell 1975a \\
\hline Pannell, Raymond & Harvest & Pannell, Beverly & Pannell 1980 \\
\hline Pannell, Raymond & Luck of Ginger Coffey, The & $\begin{array}{l}\text { Hambleton, Ronald (based on Brian } \\
\text { Moore) }\end{array}$ & Pannell 1967 \\
\hline Pannell, Raymond & Midway & Pannell, Raymond & Pannell 1975b \\
\hline Pannell, Raymond & $N-E-W-S$ & Pannell, Raymond & Pannell 1977c \\
\hline
\end{tabular}


List 1: (cont'd)

\begin{tabular}{|c|c|c|c|}
\hline Composer & Title & Librettist & Preliminary Ingraham Number \\
\hline Pannell, Raymond & Push & Pannell, Raymond & Pannell 1976 \\
\hline Pannell, Raymond & Refugees & Pannell, Beverly & Pannell 1979 \\
\hline Pannell, Raymond & $\begin{array}{l}\text { Souvenirs [As Long As a Child } \\
\text { Remembers] }\end{array}$ & Pannell, Beverly & Pannell 1978, rev. 1984 \\
\hline Parker, Michael & Visitor, The & Steffler, John & Parker 1999 \\
\hline Patenaude, Gilbert & Chevalier de Lorimier & Patenaude, Gilbert & Patenaude 1995 \\
\hline Patenaude, Gilbert & Pour ces quelques arpents de neige & Tousignant, Thérese & Patenaude 1989 \\
\hline Pelletier, Marie & Studio variazioni sull'a & Pelletier, Marie & Pelletier 1987 \\
\hline Pentland, Barbara & Lake, The & Livesay, Dorothy & Pentland 1952 \\
\hline Peters, Randolf & Golden Ass, The & Davies, Robertson & Peters 1999 \\
\hline Peters, Randolf & Inanna's Journey & Atwood, Margaret & Peters 2004 \\
\hline Peters, Randolf & Nosferatu & Gronsdal, Marilyn & Peters 1993 \\
\hline Piché, Jean & Yo soy la Desintegracion & Muckle, Yan (based on Frida Kahlo) & Piché 1997 \\
\hline Pike, Ambrose & Red Cross Princess, The & Lawrence, Ernest J. & Pike 1910 \\
\hline Polgar, Tibor & European Lover, A & Jonas, George & Polgar 1965 \\
\hline Polgar, Tibor & Glove, The & $\begin{array}{l}\text { Jonas, George (based on Fredrick } \\
\text { Schiller) }\end{array}$ & Polgar 1973 \\
\hline Polgar, Tibor & Strange Night, $A$ & $\begin{array}{l}\text { Polgar, Tibor; Pauline Carey (based on } \\
\text { Charles Szakanyi) }\end{array}$ & Polgar 1970 \\
\hline Polgar, Tibor & Troublemaker, The & Mohacsi, Eugene (trans. George Jonas) & Polgar 1968 \\
\hline Poynter, Arthur & Birth of Our Lord, The & Poynter, Arthur & Poynter 1959 \\
\hline Poynter, Arthur & Triumph of Our Lord, The & Poynter, Arthur & Poynter 1950 \\
\hline Provost, Serge & Adorable verrotière, $L^{\prime}$ & after Claude Gavreau & Provost 1992 \\
\hline
\end{tabular}




\begin{tabular}{|c|c|c|c|}
\hline Composer & Title & Librettist & Preliminary Ingraham Number \\
\hline Provost, Serge & Phaedra & Provost, Serge (based on Racine) & Provost 1987 \\
\hline Provost, Serge & Vampire et la nymphomane, Le & Gauvreau, Claude & Provost 1996 \\
\hline Quesnel, Joseph; Ridout, Godfrey & Colas et Colinette (reconstruction) & Quesnel, Joseph & Quesnel / Ridout 1963 \\
\hline Rae, Allan & C.3.3. & Rae, Allan & Rae 1987 \\
\hline Rae, Allan & Like Gods Among Them & Jennings, Fabian & Rae 1973 \\
\hline Raum, Elizabeth & Eos, The Dream of Nicholas Flood Gavin & Raum, Elizabeth; Fitzpatrick, James & Raum 1990 \\
\hline Raum, Elizabeth & Final Bid, The & Raum, Elzabeth & Raum 1980 \\
\hline Raum, Elizabeth & Garden of Alice, The & Raum, Elzabeth (based on Lewis Caroll) & Raum 1983 \\
\hline Raum, Elizabeth & Thunder in a Concave Vacuum & Raum, Elzabeth & Raum 1986 \\
\hline Rea, John & Prisoner's Play, The & Woodruff, Paul & Rea 1973 \\
\hline Renaud, Emiliano & Djymko & Renaud, Emiliano & Renaud 1926 \\
\hline Richardson, Abigail & Illusion, The & $\begin{array}{l}\text { Richardson, Abigail (based on Tony } \\
\text { Kushner, Pierre Corneille) }\end{array}$ & Richardson 2000 \\
\hline Richardson, Abigail & Mother Everest & Chan, Majorie & Richardson 2004 \\
\hline Richardson, Abigail & Seven Stories & Panych, Morris & Richardson 2002 \\
\hline Rickard, Sylvia & Fletcher's Challenge & Rickard, Sylvia & Rickard 1993 \\
\hline Ridout, Godfrey & Lost Child, The & Reid, John & Ridout 1976 \\
\hline Riley, Doug & Mandragola & Gordon, Alan & Riley 1977 \\
\hline Rogers, Stan & So Hard to Be So Hard & Rogers, Stan & Rogers 1975 \\
\hline Rolfe, James & Aenaes and Dido & Alexis, Andre & Rolfe 2007 \\
\hline
\end{tabular}


List 1: (cont'd)

\begin{tabular}{|c|c|c|c|}
\hline Composer & Title & Librettist & Preliminary Ingraham Number \\
\hline Rolfe, James & Beatrice Chancey & Clarke, George Elliot & Rolfe 1996 \\
\hline Rolfe, James & Charlotte & Podeswa, Jeremy & Rolfe $2004 a$ \\
\hline Rolfe, James & Elijah's Kite & Chai, Camyar & Rolfe 2006b \\
\hline Rolfe, James & Orpheus and Eurydice & Alexis, Andre & Rolfe $2004 b$ \\
\hline Rolfe, James & Rosa & Chai, Camyar & Rolfe $2004 c$ \\
\hline Rolfe, James & Swoon & Chatterton, Anna & Rolfe 2006a \\
\hline Ryan, George & Ghost in the Turrett, The & Grant-Lavell, Phyllis & Ryan 2003 \\
\hline Ryan, Jeffrey & Laurels, The & MacLennnan, Michael Lewis & Ryan 2002 \\
\hline Saint-Marcoux, Micheline Coulombe & Transit & Théoret, France & Saint-Marcoux 1984 \\
\hline Schafer, R. Murray & Apocalypsis, part 1: John's Vision & Schafer, R. Murray & Schafer 1977 \\
\hline Schafer, R. Murray & Apocalypsis, part 2: Credo & $\begin{array}{l}\text { Schafer, R. Murray (adapted from } \\
\text { Giordano Bruno) }\end{array}$ & Schafer 1976 \\
\hline Schafer, R. Murray & Beauty and the Beast & $\begin{array}{l}\text { Schafer, R. Murray (after Leprince de } \\
\text { Beaumont) }\end{array}$ & Schafer $1979 a$ \\
\hline Schafer, R. Murray & Characteristics Man, The & Schafer, R. Murray & Schafer 1974 \\
\hline Schafer, R. Murray & In Search of Zoroaster & Schafer, R. Murray & Schafer 1971 \\
\hline Schafer, R. Murray & Jonah & Schafer, R. Murray & Schafer 1979b \\
\hline Schafer, R. Murray & $\begin{array}{l}\text { Patria 1: Wolfman (formerly, The } \\
\text { Characteristics Man) }\end{array}$ & Schafer, R. Murray & Schafer $1987 a$ \\
\hline Schafer, R. Murray & Patria 10: The Spirit Garden Part I: Spring & Schafer, R. Murray & Schafer 1997 \\
\hline Schafer, R. Murray & $\begin{array}{l}\text { Patria 10: The Spirit Garden Part II: } \\
\text { Autumn }\end{array}$ & Schafer, R. Murray & Schafer 2001b \\
\hline
\end{tabular}




\begin{tabular}{|c|c|c|c|}
\hline Composer & Title & Librettist & Preliminary Ingraham Number \\
\hline Schafer, R. Murray & Patria 3: The Greatest Show & Schafer, R. Murray & Schafer 1987b \\
\hline Schafer, R. Murray & Patria 5: The Crown of Ariadne & $\begin{array}{l}\text { Schafer, R. Murray (from Greek mythol- } \\
\text { ogy, Epimenides) }\end{array}$ & Schafer 1991 \\
\hline Schafer, R. Murray & Patria 7: Asterion & Schafer, R. Murray & Schafer n.d. Asterion \\
\hline Schafer, R. Murray & Patria 8: The Palace of Cinnabar Phoenix & Schafer, R. Murray & Schafer 2001a \\
\hline Schafer, R. Murray & Patria 9: The Enchanted Forest & Schafer, R. Murray & Schafer 1994 \\
\hline Schafer, R. Murray & $\begin{array}{l}\text { Patria Epilogue: And Wolf Shall Inherit } \\
\text { the Moon }\end{array}$ & Schafer, R. Murray & Schafer 1988b \\
\hline Sharman, Rodney & Elsewhereless & Egoyan, Atom & Sharman 1998 \\
\hline Shepherd, Stu & $\begin{array}{l}\text { Hood of the Woods Meets the Dark Lady } \\
\text { of the Sonata, The }\end{array}$ & $\begin{array}{l}\text { Shepherd, Stu (based on Shakespeare } \\
\text { and the I Ching) }\end{array}$ & Shepherd 1984 \\
\hline Shepherd, Stu & Serrure, La & Shepherd, Stu (based on Voltaire) & Shepherd 1989 \\
\hline Sirulnikoff, Jack & This Evening & Shapli,Omar & Sirulnikoff 1960 \\
\hline Smith, Linda Catlin & Facing South & Hannah, Don & Smith 2003 \\
\hline Sokolovic, Ana & Midnight Court Opera, The & Bentley, Paul & Sokolovic 2005 \\
\hline
\end{tabular}


List 1: (cont'd)

\begin{tabular}{|c|c|c|c|}
\hline Composer & Title & Librettist & Preliminary Ingraham Number \\
\hline Somers, Harry & Death of Enkidu & Kinch, Martin & Somers 1977 \\
\hline Somers, Harry & Mario and the Magician & $\begin{array}{l}\text { Anderson, Rodney (based on Thomas } \\
\text { Mann) }\end{array}$ & Somers 1991a \\
\hline Somers, Harry & Midwinter Night's Dream, A & Wynne-Jones, Tim & Somers 1991b \\
\hline Somers, Harry & Serinette & Reaney, James & Somers 1988 \\
\hline Somers, Harry & Fool, The; Le Fou & Fram, Michael; Francoeur, Cyrille-Gauvin & Somers 1953 \\
\hline Somers, Harry & Homeless Ones, The & Fram, Michael & Somers 1955 \\
\hline Somers, Harry & Louis Riel & Moore, Mavor & Somers 1967 \\
\hline Spencer, Herbert & Cavaliers, The & Tremayne, W.A. & Spencer 1930 \\
\hline Staniland, Andrew & Ashlike on the Cradle of the Wind & Battson, Jill & Staniland 2005 \\
\hline Stone, Frank & Sardianopolis & unknown & Stone ca. 1899 \\
\hline Sullivan, Timothy & Dream Play & $\begin{array}{l}\text { Sullivan, Timothy (based on August } \\
\text { Strindberg) }\end{array}$ & Sullivan 1988 \\
\hline Sullivan, Timothy & Florence & McPherson, Anne & Sullivan 1991 \\
\hline Sullivan, Timothy & Tomorrow and Tomorrow & Sullivan, Timothy & Sullivan 1987 \\
\hline Symonds, Norman & $\begin{array}{l}\text { Charnisay Versus La Tour or, The Spirit } \\
\text { of Fundy }\end{array}$ & Symonds, Norman & Symonds 1971 \\
\hline Symonds, Norman & Episode at Big Quill & Symonds, Norman & Symonds 1978 \\
\hline Symonds, Norman & Lady in the Night & Alianak, Hrant & Symonds 1977 \\
\hline Symonds, Norman & Opera for Six Voices & Reeves, John & Symonds 1961 \\
\hline Telgmann, Oscar F. & Leo, The Royal Cadet & Cameron, George F. & Telgmann 1889 \\
\hline Thibault, Alain & Ne blâmez jamais les bédouins & Dubois, René-Daniel & Thibault 1991 \\
\hline Tremblay, Amédée & Intransigeant, L' & Tremblay, Remi & Tremblay 1906 \\
\hline
\end{tabular}




\begin{tabular}{|c|c|c|c|}
\hline Composer & Title & Librettist & Preliminary Ingraham Number \\
\hline Truax, Barry & Powers of Two: The Artist & Truax, Barry & Truax 2006 \\
\hline Turner, Robert & Brideship, The & Woodcock, George & Turner 1967 \\
\hline Turner, Robert & Vile Shadows & Newton, Norman & Turner 1982 \\
\hline Underhill, Owen & Star Catalogues, The & Diamond, Mark & Underhill 1994 \\
\hline Uyeda, Leslie & Game Misconduct & Cone, Tom & Uyeda 2000 \\
\hline Vallerand, Jean & Magicien, Le & Vallerand, Jean & Vallerand 1961 \\
\hline Vézina, Joseph & Fétiche, Le & Villandra, Alex; Fleur, Louis & Vézina 1912 \\
\hline Vézina, Joseph & Lauréat, Le & Marchand, F.G. & Vézina 1906 \\
\hline Vézina, Joseph & Rajah, Le & Michaud, B. & Vézina 1910 \\
\hline Vivier, Claude & Kopernikus & Vivier, Claude & Vivier 1979 \\
\hline Voyer, J. Ulric & Duchesse en sabots, La & $\begin{array}{l}\text { Voyer, J. Ulric (based on Ch. Le Roy- } \\
\text { Villars) }\end{array}$ & Voyer 1920 \\
\hline Voyer, J. Ulric & Jean-Marie & Voyer, J. Ulric; Rousseau, Alfred & Voyer 1923a \\
\hline Voyer, J. Ulric & Petit-Moulin & Voyer, J. Ulric & Voyer 1923b \\
\hline Voyer, J. Ulric & Intendant Bigot, L. & Voyer, J. Ulric; Rousseau, Alfred & Voyer 1928 \\
\hline Voyer, J. Ulric & Mademoiselle de Lanaudière & Voyer, J. Ulric; Deyglun, Henri & Voyer 1935 \\
\hline Wallace, Frederick William & Bound for the Rio Grande & based on English Sea Shanties & Wallace 1929 \\
\hline White, Arthur F. & Ottoman, The & Tibbs, Horace S. & White 1904 \\
\hline Wiens, Rainer & Down Here on Earth & Ward, Victoria & Wiens 1998 \\
\hline Willan, Healey & Ayreshire Ploughman, The & Willan, Healey & Willan 1930 \\
\hline
\end{tabular}


List 1: (cont'd)

\begin{tabular}{|c|c|c|c|}
\hline Composer & Title & Librettist & Preliminary Ingraham Number \\
\hline Willan, Healey & Beggar's Opera, Mr. Gay's The & Coulter, Jean (based on John Gay) & Willan 1927 \\
\hline Willan, Healey & Brébeuf & Pratt, Edwin John & Willan 1943 \\
\hline Willan, Healey & Deirdre of the Sorrows & Coutler, John & Willan 1946, rev. 1965 \\
\hline Willan, Healey & Indian Christmas [Nativity] Play, An & Ramsay, Alexander; Gibbon, John Murray & Willan 1929a \\
\hline Willan, Healey & $\begin{array}{l}\text { Ordre de Bon Temps, L'; The Order of } \\
\text { Good Cheer }\end{array}$ & $\begin{array}{l}\text { Montigny, Louvigny de (trans. J. M. } \\
\text { Gibbon) }\end{array}$ & Willan 1928 \\
\hline Willan, Healey & Prince Charlie and Flora & Gibbon, John Murray & Willan 1929b \\
\hline Willan, Healey & Transit Through Fire: An Odyssey of 1942 & Coulter, John & Willan 1942 \\
\hline Willan, Healey; Gibbon John Murray & Christmas with Herrick & $\begin{array}{l}\text { Key, John Eustace (based on Robert } \\
\text { Herrick) }\end{array}$ & Willan, Gibbon 1929 \\
\hline Wilson, Charles M. & Héloise and Abélard & Benson, Eugene & Wilson $1972 \mathrm{a}$ \\
\hline Wilson, Charles M. & Psycho Red & Benson, Eugene & Wilson 1977 \\
\hline Wilson, Charles M. & Selfish Giant, The & based on Oscar Wilde & Wilson $1972 b$ \\
\hline Wilson, Charles M. & Kamouraska & Wilson, Charles (based on Anne Hébert) & Wilson 1975 \\
\hline Wilson, Charles M. & Summoning of Everyman, The & $\begin{array}{l}\text { Benson, Eugene (based on a 14th cen- } \\
\text { tury morality play) }\end{array}$ & Wilson 1972c \\
\hline Wuensch, Gerhard & Amadeus Revisited & based on Goethe & Wuensch 1980 \\
\hline
\end{tabular}




\begin{tabular}{|c|c|}
\hline Title & Preliminary Ingraham Number \\
\hline 120 Songs for the Marquis de Sade & Hannan 2002 \\
\hline $\begin{array}{l}\text { The 90th on Active Service or, Campaigning in the } \\
\text { North West }\end{array}$ & Broughall 1885 \\
\hline Aberfan & Pannell 1977a \\
\hline Abracadabra & Freedman 1979 \\
\hline The Admiral & Harriss 1902 \\
\hline L'adorable verrotière & Provost 1992 \\
\hline Aenaes and Dido & Rolfe 2007 \\
\hline Airline Icarus & Current 2006a \\
\hline Alice and Friends & Evangelista 1990 \\
\hline All for Love (see Nancy) & Aldous $1902 b$ \\
\hline Altar of the Sun & Burritt 1983 \\
\hline Amadeus Revisited & Wuensch 1980 \\
\hline The Animals of Limbo & Pannell 1990 \\
\hline Amy Rosbart & Horner n.d Amy \\
\hline Angel Square & Crawley 1996 \\
\hline Anne Hathaway & Lucas 1887 \\
\hline Apocalypsis, part 1: John's Vision & Schafer 1977 \\
\hline Apocalypsis, part 2: Credo & Schafer 1976 \\
\hline L'appel du missionnaire & Gingras 1925 \\
\hline Arabia & Lucas 1886 \\
\hline The Architect & MacIntyre 1994 \\
\hline Aria da Capo & Pannell 1963 \\
\hline The Aristocratic Lover & Arsenault 1987 \\
\hline Around Alline & Miller 1989 \\
\hline The Artist (see Powers of Two: The Artist) & Truax 2006 \\
\hline As Long As a Child Remembers (see Souvenirs) & Pannell 1978, rev. 1984 \\
\hline Ashlike on the Cradle of the Wind & Staniland 2005 \\
\hline Asks Alice & Hatch 1998 \\
\hline Asterion (see Patria 7) & Schafer n.d. Asterion \\
\hline Autumn (see Patria 10: The Spirit Garden Part II) & Schafer $2001 b$ \\
\hline The Ayreshire Ploughman & Willan 1930 \\
\hline Ballade du fils de l'homme & Charpentier n.d. Ballade \\
\hline The Bargain & McPeek 1963 \\
\hline
\end{tabular}


List 2: (cont'd)

\begin{tabular}{|c|c|}
\hline Title & Preliminary Ingraham Number \\
\hline Barnardo Boy & Crawley 1980 \\
\hline Beatrice Chancey & Rolfe 1996 \\
\hline Beauty and the Beast & Schafer 1979a \\
\hline The Beggar's Opera, Mr. Gay's & Willan 1927 \\
\hline La Belle et la Bête & Duncan 2004 \\
\hline The Bells of Barcelona & Horner 1911 \\
\hline Beowulf & Davies 1974 \\
\hline Beware of the Wolf & Detweiler 1964 \\
\hline The Birth of Our Lord & Poynter 1959 \\
\hline The Birthday of the Infanta & Farrell 1979 \\
\hline $\begin{array}{l}\text { The Black Theatre of Hermes Trismegistos (see } \\
\text { Patria 4) }\end{array}$ & Schafer 1988a \\
\hline Boiler Room Suite & Doolittle 1988 \\
\hline Bones & Buck 2001 \\
\hline Bound for the Rio Grande & Wallace 1929 \\
\hline Le Bourgeois gentilhomme & Charpentier 1964 \\
\hline Brébeuf & Willan 1943 \\
\hline The Brideship & Turner 1967 \\
\hline The Broken Ring & Jones 1953 \\
\hline The Brothers Grimm & Burry 2001 \\
\hline $\begin{array}{l}\text { Bunthorne Abroad or, The Lass That Loved a } \\
\text { Pirate }\end{array}$ & Bengough 1883 \\
\hline Burnt Toast: 8 mini comic operas about love & Louie 2006 \\
\hline Byron, the Wonderful Bandit & Barnes 1965 \\
\hline C.3.3. & Rae 1987 \\
\hline $\begin{array}{l}\text { Campaigning in the North West (see The 90th on } \\
\text { Active Service) }\end{array}$ & Broughall 1885 \\
\hline Canada's Welcome: A Masque & Clappé 1879 \\
\hline A Canadian Carnival (see Ptarmigan) & Aldous 1895 \\
\hline Carmina Tenebrarum & Keane 1983 \\
\hline The Cavaliers & Spencer 1930 \\
\hline The Cellar Door & Hui 2002 \\
\hline Ceyx and Alcyone & Kunz 1979 \\
\hline The Characteristics Man (see also Patria 1) & Schafer 1974 \\
\hline Charlie the Chicken & Doolittle 1975 \\
\hline Charlotte & Rolfe $2004 a$ \\
\hline
\end{tabular}


List 2: (cont'd)

\begin{tabular}{|c|c|}
\hline Title & Preliminary Ingraham Number \\
\hline Charnisay Versus La Tour or, The Spirit of Fundy & Symonds 1971 \\
\hline Chevalier de Lorimier & Patenaude 1995 \\
\hline Christmas with Herrick & Willan, Gibbon 1929 \\
\hline Cingalee & Doyle ca. 1915 \\
\hline Circe & Pannell 1977b \\
\hline $\begin{array}{l}\text { Clara et les philosophes; Clara and the } \\
\text { Philosophers }\end{array}$ & Charpentier 1976 \\
\hline Clara on the Bridge & Charpentier n.d. Clara Bridge \\
\hline Clara, la nuit & Charpentier n.d. Clara Nuit \\
\hline Clara, un masque & Charpentier n.d. Clara masque \\
\hline Clarabelle-Clarimage & Charpentier 1979 \\
\hline Claracello ou Répertoire & Charpentier 1985 \\
\hline Clara-Committee & Charpentier n.d. Clara Committee \\
\hline Clara-Teacher (see An English Lesson) & Charpentier 1968 \\
\hline Clear Sky \& Thunder & Henderson 1983 \\
\hline Colas et Colinette (reconstruction) & Quesnel / Ridout 1963 \\
\hline The Concrete Rose & Goldberg 1970 \\
\hline Constantinople & Hatzis 2004 \\
\hline $\begin{array}{l}\text { La Conversion d'un pêcheur de la Nouvelle- } \\
\text { Écosse }\end{array}$ & Labelle 1884 \\
\hline Coup de Soleil & Létourneau 1930 \\
\hline Crazy to Kill & Beckwith 1988 \\
\hline Credo (see Apocalypsis, part 2) & Schafer 1976 \\
\hline The Crown of Ariadne (see Patria 5) & Schafer 1991 \\
\hline The Curfew & Kerrison $1879 a$ \\
\hline The Damask Drum & Kunz 1962a \\
\hline The Death of a Hired Man & Mclntyre 1966a \\
\hline Death of Enkidu & Somers 1977 \\
\hline $\begin{array}{l}\text { Demise of Innocence (see Dulcitius: Demise of } \\
\text { Innocence) }\end{array}$ & Koprowski 1989 \\
\hline Deirdre of the Sorrows & Willan 1946, rev. 1965 \\
\hline The Devil's Constructs or, The Eighth Day & Keane 1978 \\
\hline Djymko & Renaud 1926 \\
\hline Doctor Canon's Cure & Holman 1982 \\
\hline
\end{tabular}


List 2: (cont'd)

\begin{tabular}{|c|c|}
\hline Title & Preliminary Ingraham Number \\
\hline The Donkey's Tale & Mann 1971 \\
\hline Down Here on Earth & Wiens 1998 \\
\hline Drakkar & Dolin 1972 \\
\hline The Dream Healer (see Pilgrim) & Burritt 2003 \\
\hline $\begin{array}{l}\text { The Dream of Nicholas Flood Gavin (see Eos, The } \\
\text { Dream of Nicholas Flood Gavin) }\end{array}$ & Raum 1990 \\
\hline Dream Play & Sullivan 1988 \\
\hline La Duchesse en sabots & Voyer 1920 \\
\hline Dulcitius: Demise of Innocence & Koprowski 1989 \\
\hline The Eighth Day (see The Devil's Constructs) & Keane 1978 \\
\hline Electric Chair & Burritt 1971 \\
\hline Elijah's Kite & Rolfe $2006 b$ \\
\hline Elsewhereless & Sharman 1998 \\
\hline The Enchanted Forest (see Patria 9) & Schafer 1994 \\
\hline The Enchanted Garden & Doyle 1916 \\
\hline Les Enfants du manoir & Lavigueur 1881a \\
\hline An English Lesson or, Clara-Teacher & Charpentier 1968 \\
\hline Eos, The Dream of Nicholas Flood Gavin & Raum 1990 \\
\hline Episode at Big Quill & Symonds 1978 \\
\hline Erehwon & Applebaum 2000 \\
\hline Les Étrivières & Frenette 1983 \\
\hline A European Lover & Polgar 1965 \\
\hline Evangeline & George 1948 \\
\hline Évangéline et Gabriel & Gagné 1994 \\
\hline $\begin{array}{l}\text { Exercices de conversation et de diction françaises } \\
\text { pour étudiants américains }\end{array}$ & Évangelista 1992 \\
\hline Exiles & Pannell 1973 \\
\hline An Expensive Embarrassment & Gougeon 1989 \\
\hline Eyes on the Mountain & Donison 2001 \\
\hline Facing South & Smith 2003 \\
\hline Fall of the House of Usher & Duncan 2001 \\
\hline Le Fétiche & Vézina 1912 \\
\hline La Fiancée des bois & Lavigueur 1881b \\
\hline Filumena & Estacio 2003 \\
\hline The Final Bid & Raum 1980 \\
\hline
\end{tabular}


List 2: (cont'd)

\begin{tabular}{|c|c|}
\hline Title & Preliminary Ingraham Number \\
\hline Fletcher's Challenge & Rickard 1993 \\
\hline Florence & Sullivan 1991 \\
\hline The Fool; Le Fou & Somers 1953 \\
\hline $\begin{array}{l}\text { Forestiers et Voyageurs (see Scènes des } \\
\text { Voyageurs) }\end{array}$ & O'Brien 1928 \\
\hline Francine & Morin-Labrecque 1930 \\
\hline Frobisher & Estacio 2006 \\
\hline From The [Buczynski] Book of the Living & Buczynski 1972 \\
\hline Galatea elettronica & Goldberg 1969 \\
\hline Game Misconduct & Uyeda 2000 \\
\hline The Gandy Dance & Faith 1943 \\
\hline The Gang & Hannan 1999 \\
\hline The Garden of Alice & Raum 1983 \\
\hline Ghost Dance & Levin 1985 \\
\hline The Ghost in the Turrett & Ryan 2003 \\
\hline The Girl of Bandolier & Leonard 1952 \\
\hline Gisèle & Lavallée-Smith 1911 \\
\hline The Glove & Polgar 1973 \\
\hline Go & Pannell 1975a \\
\hline The Golden Age & Doyle 1915 \\
\hline The Golden Ass & Peters 1999 \\
\hline A Golden Catch & Aldous $1902 a$ \\
\hline The Grandee & Hone 1899 \\
\hline Grant, Warden of the Plains & Adaskin 1967 \\
\hline The Greatest Show (see Patria 3) & Schafer $1987 b$ \\
\hline Guacamayo's Old Song and Dance & Oliver 1991 \\
\hline $\begin{array}{l}\text { Halifax to the Saskatchewan: 'Our Boys' in the Riel } \\
\text { Rebellion }\end{array}$ & Dixon 1886 \\
\hline Hamlet, Prince of Dry Goods (see Puffe \& Co.) & Lucas 1889 \\
\hline Hannaraptor & Gilliland 2007 \\
\hline Harvest & Pannell 1980 \\
\hline The Hay Makers & Nelson 1869 \\
\hline Héloise and Abélard & Wilson $1972 a$ \\
\hline His Majesty's Pie & Bissell 1966 \\
\hline $\begin{array}{l}\text { HMS Parliament or, The Lady Who Loved a } \\
\text { Government Clerk }\end{array}$ & Fuller 1879 \\
\hline
\end{tabular}


List 2: (cont'd)

\begin{tabular}{|c|c|}
\hline Title & Preliminary Ingraham Number \\
\hline The Hobbit & Burry 2004 \\
\hline The Homeless Ones & Somers 1955 \\
\hline $\begin{array}{l}\text { The Hood of the Woods Meets the Dark Lady of } \\
\text { the Sonata }\end{array}$ & Shepherd 1984 \\
\hline Humulus the Mute & MacIntyre 1977 \\
\hline Hypnos & Ford 1972 \\
\hline Ice Time & Chan 2004 \\
\hline The Illusion & Richardson 2000 \\
\hline In Search of Zoroaster & Schafer 1971 \\
\hline Inanna's Journey & Peters 2004 \\
\hline An Indian Christmas [Nativity] Play & Willan 1929a \\
\hline Inook & Bottenberg 1986 \\
\hline L'Intendant Bigot & Voyer 1928 \\
\hline L'Intransigeant & Tremblay 1906 \\
\hline Inventory & Current 2006b \\
\hline Iron Road & Chan 2000 \\
\hline Isis and the Seven Scorpions & Burry 2006a \\
\hline Island & Donison 2006 \\
\hline Jean-Marie & Voyer 1923a \\
\hline John's Vision (see Apocalypsis, part 1) & Schafer 1977 \\
\hline The Jolly Beggars & Gibbon 1928 \\
\hline Jonah & Schafer 1979b \\
\hline Le Jugement de Salomon & Lavallée 1886 \\
\hline Kamouraska & Wilson 1975 \\
\hline A King for Corsica & George 1975 \\
\hline Klondyke & Charpentier 1965 \\
\hline Kopernikus & Vivier 1979 \\
\hline Lady Blancarosa & Komorous 1966 \\
\hline Lady Diotima's Walk (see Millenial Mall) & Anhalt 2000 \\
\hline Lady in the Night & Symonds 1977 \\
\hline $\begin{array}{l}\text { The Lady Who Loved a Government Clerk (see } \\
\text { HMS Parliament) }\end{array}$ & Fuller 1879 \\
\hline Lagalai-Legend of the Stone & Kenins 1970 \\
\hline The Lake & Pentland 1952 \\
\hline The Land of the Maple Leaf (see La Terre Bonne) & Geddes-Harvey 1903 \\
\hline
\end{tabular}


List 2: (cont'd)

\begin{tabular}{|c|c|}
\hline Title & Preliminary Ingraham Number \\
\hline $\begin{array}{l}\text { The Lass That Loved a Pirate (see Bunthorne } \\
\text { Abroad) }\end{array}$ & Bengough 1883 \\
\hline The Last Duel & Kulesha 1999 \\
\hline The Last of the Aztecs & Kerrison 1914 \\
\hline Le Lauréat & Vézina 1906 \\
\hline The Laurels & Ryan 2002 \\
\hline The Lay of Trym & Behrens 1968 \\
\hline La Légende du vent & Benoist 1974 \\
\hline Leo, The Royal Cadet & Telgmann 1889 \\
\hline Let Us Pay Tribute to Lord Gordon & Davies 1968 \\
\hline Let's Make a Carol & Kunz 1965a \\
\hline Like Gods Among Them & Rae 1973 \\
\hline Lisa & Daniel 2002 \\
\hline Lolita & Miro 1922 \\
\hline Long March/Shining Path (see Trudeau) & Jackson 2007 \\
\hline The Lost Child & Ridout 1976 \\
\hline Louis Riel & Somers 1967 \\
\hline Loulou & Lavallée 1872 \\
\hline Loving (see Tol) & Schafer 1965 \\
\hline The Loyalists & Major 1967 \\
\hline The Luck of Ginger Coffey & Pannell 1967 \\
\hline Madamoiselle Fifi & Hannah 1983 \\
\hline Mademoiselle de Lanaudière & Voyer 1935 \\
\hline Le Magicien & Vallerand 1961 \\
\hline The Maid of the Mill & Kerrison $1879 b$ \\
\hline Maids of Hamelin & Leonard 1943 \\
\hline The Maire of St. Brieux & Mills 1875 \\
\hline Mam'zell Bebe & Létourneau 1933 \\
\hline Mandragola & Riley 1977 \\
\hline Manuscrit trouvé à Saragosse & Evangelista 2001 \\
\hline Un Mariage improvisé & Lavigueur 1881c \\
\hline Mariken of Nimmegen & Danielson 2003 \\
\hline Mario and the Magician & Somers 1991a \\
\hline Master Logdriver (see Menaud) & Gagné 1986 \\
\hline The Meal & Archer 1983 \\
\hline
\end{tabular}


List 2: (cont'd)

\begin{tabular}{|c|c|}
\hline Title & Preliminary Ingraham Number \\
\hline Menaud (Master Logdriver) & Gagné 1986 \\
\hline Une mesure de silence; A Silent Measure & Blackburn 1954 \\
\hline The Midnight Court Opera & Sokolovic 2005 \\
\hline Midway & Pannell 1975b \\
\hline A Midwinter Night's Dream & Somers 1991b \\
\hline Millenial Mall (Lady Diotima's Walk) & Anhalt 2000 \\
\hline A Million Dollar Girl; Le petit Milliardaire & Miro 1915 \\
\hline Mira & Lamarche 1981 \\
\hline The Miraculous Turnip & Bissell 1980 \\
\hline The Money Spider & Lucas 1897 \\
\hline Mother Everest & Richardson 2004 \\
\hline $\begin{array}{l}\text { Mr. Gay's The Beggar's Opera (see The Beggar's } \\
\text { Opera) }\end{array}$ & Willan 1927 \\
\hline A Musical Play & Bissell 1977 \\
\hline Naked at the Opera & Buczynski 1978 \\
\hline Nancy or, All for Love & Aldous $1902 b$ \\
\hline Naomi's Road & Luengen 2005 \\
\hline Ne blâmez jamais les bédouins & Thibault 1991 \\
\hline Nelligan & Gagnon 1990 \\
\hline$N-E-W-S$ & Pannell 1977c \\
\hline Night Blooming Cereus & Beckwith 1958 \\
\hline Nightbloom & Jaeger, Lake, Montgomery 1984 \\
\hline Nigredo Hotel & Gotham 1992 \\
\hline No No Miya & Komorous 1988 \\
\hline The Nobleman's Wedding & Morgan 1982 \\
\hline Une noce canadienne-française & O’Brien 1930a \\
\hline Nosferatu & Peters 1993 \\
\hline An Odyssey of 1942 (see Transit Through Fire) & Willan 1942 \\
\hline Onadéga ou la tragédie de lac des bois & Benoist 1977 \\
\hline Once Again . . . Pop! & Burritt 1969 \\
\hline Opera for Six Voices & Symonds 1961 \\
\hline Opera on the Rocks & Ogborn 2007 \\
\hline OPéRAaaAH! & Deschênes 1983 \\
\hline L'Ordre de Bon Temps; The Order of Good Cheer & Willan 1928 \\
\hline Oreads & Kerrison 1870 \\
\hline
\end{tabular}


List 2: (cont'd)

\begin{tabular}{|c|c|}
\hline Title & Preliminary Ingraham Number \\
\hline Orphée I & Charpentier 1969 \\
\hline Orphée II & Charpentier $1972 a$ \\
\hline Orpheus & Klein 1976 \\
\hline Orpheus and Eurydice & Rolfe 2004b \\
\hline Oscar Excerpts: The Picture of Dorian Gray & Luedeke 1996 \\
\hline The Ottoman & White 1904 \\
\hline L'Ouï-dire & Hambraeus 1986 \\
\hline $\begin{array}{l}\text { 'Our Boys' in the Riel Rebellion (see Halifax to the } \\
\text { Saskatchewan) }\end{array}$ & Dixon 1886 \\
\hline Over the Japanese Sea & Palmer 2003 \\
\hline The Palace of Cinnabar Phoenix (see Patria 8) & Schafer 2001a \\
\hline $\begin{array}{l}\text { Patria 1: Wolfman (formerly, The Characteristics } \\
\text { Man) }\end{array}$ & Schafer $1987 a$ \\
\hline Patria 10: The Spirit Garden Part I: Spring & Schafer 1997 \\
\hline Patria 10: The Spirit Garden Part II: Autumn & Schafer 2001b \\
\hline Patria 2: Requiems for the Party Girl & Schafer 1972 \\
\hline Patria 3: The Greatest Show & Schafer 1987b \\
\hline $\begin{array}{l}\text { Patria 4: The Black Theatre of Hermes } \\
\text { Trismegistos }\end{array}$ & Schafer 1988a \\
\hline Patria 5: The Crown of Ariadne & Schafer 1991 \\
\hline Patria 6: RA & Schafer 1983 \\
\hline Patria 7: Asterion & Schafer n.d. Asterion \\
\hline Patria 8: The Palace of Cinnabar Phoenix & Schafer 2001a \\
\hline Patria 9: The Enchanted Forest & Schafer 1994 \\
\hline Patria Epilogue: And Wolf Shall Inherit the Moon & Schafer 1988b \\
\hline Patria: The Princess of Stars: The Prologue & Schafer 1981 \\
\hline Peggy Machree & Lucas 1904 \\
\hline Le Père des Amours & Lapierre 1942 \\
\hline The Perils of Clara (see The Tea Symphony) & Charpentier $1972 b$ \\
\hline Petit-Moulin & Voyer 1923b \\
\hline Phaedra & Provost 1987 \\
\hline Philippino & O'Brien 1933 \\
\hline The Picture of Dorian Gray (see Oscar Excerpts) & Luedeke 1996 \\
\hline The Pied Piper & Crawley 1989 \\
\hline Pilgrim [The Dream Healer] & Burritt 2003 \\
\hline Pipandor & Harrison 1884 \\
\hline
\end{tabular}


List 2: (cont'd)

\begin{tabular}{|c|c|}
\hline Title & Preliminary Ingraham Number \\
\hline Pirouette & Blackburn 1960 \\
\hline Portrait of a City & Harris 1952 \\
\hline The Poster Girl & Aldous $1902 \mathrm{c}$ \\
\hline Pour ces quelques arpents de neige & Patenaude 1989 \\
\hline Powers of Two: The Artist & Truax 2006 \\
\hline Prince Charlie and Flora & Willan 1929b \\
\hline Prince Charming & MacMillan 1931 \\
\hline Prince Tommy & Delaney 1898 \\
\hline La Princesse blanche & Mather 1993 \\
\hline $\begin{array}{l}\text { The Princess of Stars: The Prologue (see Patria: } \\
\text { The Princess of the Stars) }\end{array}$ & Schafer 1981 \\
\hline The Prisoner's Play & Rea 1973 \\
\hline Le Prix & Hétu 1992 \\
\hline Psycho Red & Wilson 1977 \\
\hline Ptarmigan or, A Canadian Carnival & Aldous 1895 \\
\hline Puffe \& Co. or, Hamlet, Prince of Dry Goods & Lucas 1889 \\
\hline Push & Pannell 1976 \\
\hline Québécité & Jackson 2003 \\
\hline The Queen of Romance & Higgin 1946 \\
\hline The Queen's Shilling & Maclagan 1880 \\
\hline RA (see Patria 6) & Schafer 1983 \\
\hline Le Rajah & Vézina 1910 \\
\hline Réalitillusion & Brégent 1988 \\
\hline Rebel and Empire & Levin 1978 \\
\hline The Red Cross Princess & Pike 1910 \\
\hline Red Emma & Kulesha 1993 \\
\hline Refrains & MacIntyre 1981 \\
\hline Refugees & Pannell 1979 \\
\hline La Rencontre dans l'escalier & Benoist n.d. Rencontre \\
\hline Requiems for the Party Girl (see Patria 2) & Schafer 1972 \\
\hline The Return of the Native & Coulthard 1956 \\
\hline Revolt in Upper Canada & George 1987 \\
\hline Riders to the Sea & Betts 1955 \\
\hline Rip Van Winkle & Manning 1919 \\
\hline Le Roman de Suzon & Miro 1914 \\
\hline
\end{tabular}


List 2: (cont'd)

\begin{tabular}{|c|c|}
\hline Title & Preliminary Ingraham Number \\
\hline Rosa & Rolfe $2004 c$ \\
\hline Rumplestilskin & Higgin 1945 \\
\hline Sagan (The Legend) & Hambraeus 1979 \\
\hline Saint Carmen of the Main & Hodkinson 1988 \\
\hline Saint-François d'Assise & Benoist n.d. Saint \\
\hline Á Saint Malo (see Visions canadiennes) & O'Brien 1930b \\
\hline Salmon Dance & Mcley 1978, rev 1996 \\
\hline Sam Slick & Jones 1965 \\
\hline Sardianopolis & Stone ca. 1899 \\
\hline The Scarlet Princess & Louie 2002 \\
\hline Scènes des voyageurs [Forestiers et voyageurs] & O'Brien 1928 \\
\hline Scenic Mosaics & Badian 1997 \\
\hline Scottish Landing & Jones 1967 \\
\hline Seabird Island & Healey 1977 \\
\hline The Second Shepherds' Play & Doolittle 1980 \\
\hline Le Secret des Amati & Benoist n.d. Secret \\
\hline The Selfish Giant & Cabena 1970 \\
\hline The Selfish Giant & Wilson $1972 b$ \\
\hline Semiramis & Lucas 1884 \\
\hline Serinette & Somers 1988 \\
\hline La Serrure & Shepherd 1989 \\
\hline Seven Stories & Richardson 2002 \\
\hline Sganarelle & Archer 1973 \\
\hline The Shadow & Daniel 2007 \\
\hline Shanawdithit & Burry 1996 \\
\hline Shelter & Palmer 2007 \\
\hline The Shivaree & Beckwith 1978 \\
\hline Silents! & Freedman 1978 \\
\hline Silver City & Doolittle 1984 \\
\hline The Slaughter of the Innocents & Crawley 1974 \\
\hline So Hard to Be So Hard & Rogers 1975 \\
\hline Sœur Béatrice & Laliberté 1920 \\
\hline Souvenirs [As Long As a Child Remembers] & Pannell 1978, rev. 1984 \\
\hline The Spirit Garden Part I: Spring (see Patria 10) & Schafer 1997 \\
\hline
\end{tabular}


List 2: (cont'd)

\begin{tabular}{|c|c|}
\hline Title & Preliminary Ingraham Number \\
\hline The Spirit Garden Part II: Autumn (see Patria 10) & Schafer 2001b \\
\hline $\begin{array}{l}\text { The Spirit of Fundy (see Charnisay Versus La } \\
\text { Tour) }\end{array}$ & Symonds 1971 \\
\hline Spring (see Patria 10: The Spirit Garden Part I) & Schafer 1997 \\
\hline The Star Catalogues & Underhill 1994 \\
\hline The Star-Child & Greer 2000 \\
\hline A Strange Night & Polgar 1970 \\
\hline Studio variazioni sull'a & Pelletier 1987 \\
\hline The Summoning of Everyman & Wilson 1972c \\
\hline Swoon & Rolfe 2006a \\
\hline Taptoo! & Beckwith 1993 \\
\hline The Tea Symphony or, The Perils of Clara & Charpentier 1972b \\
\hline $\begin{array}{l}\text { The Tearful and Tragical Tale of the Tricky } \\
\text { Troubadour or, The Truant Tracked }\end{array}$ & Broughall 1886 \\
\hline La Terre Bonne or, The Land of the Maple Leaf & Geddes-Harvey 1903 \\
\hline Thirteen Hands & Mclntyre 2000 \\
\hline This Evening & Sirulnikoff 1960 \\
\hline This Is Not True & McIntyre $1966 b$ \\
\hline Three Figures in a Crystal Ball & Kunz 1962b \\
\hline Thunder in a Concave Vacuum & Raum 1986 \\
\hline Tikkum (see Traces) & Anhalt 1996 \\
\hline Tis Pity & Lake 1990 \\
\hline Toi; Loving & Schafer 1965 \\
\hline Tomorrow and Tomorrow & Sullivan 1987 \\
\hline Toothpaste & Louie 2001 \\
\hline Torquil & Harriss 1896 \\
\hline La Tourangelle & Anhalt 1975 \\
\hline Traces (Tikkum) & Anhalt 1996 \\
\hline Transit & Saint-Marcoux 1984 \\
\hline Transit Through Fire: An Odyssey of 1942 & Willan 1942 \\
\hline The Traveling Musicians & Adaskin 1983 \\
\hline The Triumph of Our Lord & Poynter 1950 \\
\hline The Troublemaker & Polgar 1968 \\
\hline $\begin{array}{l}\text { The Truant Tracked (see The Tearful and Tragical } \\
\text { Tale of the Tricky Troubadour) }\end{array}$ & Broughall 1886 \\
\hline Trudeau: Long March/Shining Path & Jackson 2007 \\
\hline
\end{tabular}


List 2: (cont'd)

\begin{tabular}{|c|c|}
\hline Title & Preliminary Ingraham Number \\
\hline Turtle Wakes & Bell 2001 \\
\hline The Two Graces & Ferguson 2004 \\
\hline The Unbelievable Glory of Mr. Sharp & MacDonald 1989 \\
\hline Unto the Earth: Vignettes of a War & Burry 1993 \\
\hline Le Vagabond de la gloire & Lapierre 1947 \\
\hline Le Vampire et la nymphomane & Provost 1996 \\
\hline Les Verdi & Gagné 2001 \\
\hline La Veuve; The Widow & Lavallée 1880 \\
\hline Via Cruxus & Daniel 2004 \\
\hline Vignettes of a War (see Unto the Earth) & Burry 1993 \\
\hline Vile Shadows & Turner 1982 \\
\hline The Vinland Traveler & Burry 2006b \\
\hline Visions canadiennes: Á Saint Malo & O'Brien 1930b \\
\hline The Visitor & Parker 1999 \\
\hline Vive la Canadienne & Létourneau 1924 \\
\hline The Watchful Gods & Kunz 1965b \\
\hline Way Out & George 1960 \\
\hline Winthrop & Anhalt 1986 \\
\hline $\begin{array}{l}\text { And Wolf Shall Inherit the Moon (see Patria } \\
\text { Epilogue) }\end{array}$ & Schafer $1988 b$ \\
\hline Wolfman (see Patria 1) & Schafer $1987 a$ \\
\hline The Woodcarver's Wife & Betts 1960 \\
\hline The Woodcarver's Wife & Eggleston 1961 \\
\hline Yo soy la Desintegracion & Piché 1997 \\
\hline Zaâ le loup & Désilets 1997 \\
\hline Zaria & Désilets 1990 \\
\hline Zoé & Désilets 1988 \\
\hline Zurich 1916 & Butterfield 1998 \\
\hline
\end{tabular}




\begin{tabular}{|c|c|c|}
\hline $\begin{array}{l}\text { Preliminary } \\
\text { Ingraham } \\
\text { Number }\end{array}$ & Title & Genre \\
\hline Nelson 1869 & Hay Makers, The & Comic Opera 1 \\
\hline Kerrison 1870 & Oreads & Opera 4 \\
\hline Lavallée 1872 & Loulou & Comic Opera 1 [Opera 4] \\
\hline Mills 1875 & Maire of St. Brieux, The & Operetta 4 \\
\hline Clappé 1879 & Canada's Welcome: A Masque & Masque 4 \\
\hline Fuller 1879 & $\begin{array}{l}\text { HMS Parliament or, The Lady Who } \\
\text { Loved a Government Clerk }\end{array}$ & Operetta 1 [Burlesque 4] \\
\hline Kerrison 1879a & Curfew, The & Opera 4 \\
\hline Kerrison $1879 b$ & Maid of the Mill, The & Light Opera 4 \\
\hline Lavallée 1880 & Veuve, La; The Widow & $\begin{array}{l}\text { Comic Opera 1, } 2 \text { [Opera; Operetta 3; } \\
\text { Light Opera 4] }\end{array}$ \\
\hline Maclagan 1880 & Queen's Shilling, The & Comic Opera 1 \\
\hline Lavigueur 1881a & Enfants du manoir, Les & Comic Opera 1 \\
\hline Lavigueur $1881 \mathrm{~b}$ & Fiancée des bois, La & Operetta 1 [Light Opera 4] \\
\hline Lavigueur 1881c & Mariage improvisé, Un & Comic Opera 1 \\
\hline Bengough 1883 & $\begin{array}{l}\text { Bunthorne Abroad or, The Lass That } \\
\text { Loved a Pirate }\end{array}$ & Operetta 1 \\
\hline Harrison 1884 & Pipandor & Comic Opera 1 [Opera 4] \\
\hline Labelle 1884 & $\begin{array}{l}\text { Conversion d'un pêcheur de la } \\
\text { Nouvelle-Écosse, La }\end{array}$ & Operetta 4 \\
\hline Lucas 1884 & Semiramis & Opera 4 \\
\hline Broughall 1885 & $\begin{array}{l}\text { 90th on Active Service, The; or, } \\
\text { Campaigning in the North West }\end{array}$ & Burlesque 4 \\
\hline Broughall 1886 & $\begin{array}{l}\text { Tearful and Tragical Tale of the Tricky } \\
\text { Troubadour, The; or, The Truant } \\
\text { Tracked }\end{array}$ & Burlesque In Grand Opera Style 4 \\
\hline Dixon 1886 & $\begin{array}{l}\text { Halifax to the Saskatchewan: 'Our Boys' } \\
\text { in the Riel Rebellion }\end{array}$ & Burlesque 4 \\
\hline Lavallée 1886 & Jugement de Salomon, Le & Opera 4 \\
\hline Lucas 1886 & Arabia & Opera 4 \\
\hline Lucas 1887 & Anne Hathaway & Opera 4 \\
\hline
\end{tabular}


LIST 3: (cont'd)

\begin{tabular}{|c|c|c|}
\hline $\begin{array}{l}\text { Preliminary } \\
\text { Ingraham } \\
\text { Number }\end{array}$ & Title & Genre \\
\hline Lucas 1889 & $\begin{array}{l}\text { Puffe \& Co. or, Hamlet, Prince of Dry } \\
\text { Goods }\end{array}$ & Comic Opera 6 \\
\hline Telgmann 1889 & Leo, The Royal Cadet & Opera; Operetta 4 \\
\hline Aldous 1895 & Ptarmigan or, A Canadian Carnival & Comic Opera 4 \\
\hline Harriss 1896 & Torquil & $\begin{array}{l}\text { Scandanavian Dramatic Legend } 1 \\
\text { [Opera 4] }\end{array}$ \\
\hline Lucas 1897 & Money Spider, The & Opera 4 \\
\hline Delaney 1898 & Prince Tommy & Operetta 4 \\
\hline Hone 1899 & Grandee, The & Comic Opera 1 [Opera 4] \\
\hline Stone ca. 1899 & Sardianopolis & Opera 4 \\
\hline Aldous $1902 a$ & Golden Catch, A & Operetta 4 \\
\hline Aldous 1902b & Nancy or, All for Love & Operetta 4 \\
\hline Aldous 1902c & Poster Girl, The & Operetta 4 \\
\hline Harriss 1902 & Admiral, The & Comic Opera 1 \\
\hline $\begin{array}{l}\text { Geddes-Harvey } \\
1903\end{array}$ & $\begin{array}{l}\text { Terre Bonne, La; or, The Land of the } \\
\text { Maple Leaf }\end{array}$ & Opera 4 \\
\hline Lucas 1904 & Peggy Machree & Operetta 4 \\
\hline White 1904 & Ottoman, The & Comic Opera 1 \\
\hline Tremblay 1906 & Intransigeant, L' & Operetta 1, 4 \\
\hline Vézina 1906 & Lauréat, Le & Opéra Comique 1 [Operetta 4] \\
\hline Pike 1910 & Red Cross Princess, The & Comic Opera 1 \\
\hline Vézina 1910 & Rajah, Le & Opéra Bouffe 1 [Operetta 4] \\
\hline Horner 1911 & Bells of Barcelona, The & Operetta 4 \\
\hline Horner n.d Amy & Amy Rosbart & Opera 4 \\
\hline $\begin{array}{l}\text { Lavallée-Smith } \\
1911\end{array}$ & Gisèle & Operetta 1, 4 [Light Opera 4] \\
\hline Vézina 1912 & Fétiche, Le & Opéra Comique 1, 4 \\
\hline Kerrison 1914 & Last of the Aztecs, The & Opera 4 \\
\hline
\end{tabular}


LIST 3: (cont'd)

\begin{tabular}{|c|c|c|}
\hline $\begin{array}{l}\text { Preliminary } \\
\text { Ingraham } \\
\text { Number }\end{array}$ & Title & Genre \\
\hline Miro 1914 & Roman de Suzon, Le & Operetta 1, 4 [Light Opera; Operetta 4] \\
\hline Doyle 1915 & Golden Age, The & Comic Opera 1 [Opera 4] \\
\hline $\begin{array}{l}\text { Doyle n.d } \\
\text { Cingalee }\end{array}$ & Cingalee & Operetta 4 \\
\hline Miro 1915 & Million Dollar Girl, A; Le petit Milliardaire & Comic Opera 1 \\
\hline Doyle 1916 & Enchanted Garden, The & Comic Opera 4 \\
\hline Manning 1919 & Rip Van Winkle & American Folk Opera 2 \\
\hline Laliberté 1920 & Sœur Béatrice & Opera 1,4 \\
\hline Voyer 1920 & Duchesse en sabots, La & Comic Opera 6 \\
\hline Miro 1922 & Lolita & Operetta 1, 4 \\
\hline Voyer 1923a & Jean-Marie & Comic Opera 6 \\
\hline Voyer 1923b & Petit-Moulin & Comic Opera 6 \\
\hline Létourneau 1924 & Vive la Canadienne & Operetta 1, 4 \\
\hline Gingras 1925 & Appel du missionnaire, $L^{\prime}$ & Operetta 1 \\
\hline Renaud 1926 & Djymko & Musical Farce 1 [Opera 4] \\
\hline Willan 1927 & Beggar's Opera, Mr. Gay's The & Ballad Opera 4 \\
\hline Gibbon 1928 & Jolly Beggars, The & Ballad Opera 6 \\
\hline O'Brien 1928 & $\begin{array}{l}\text { Scènes des voyageurs [Forestiers et } \\
\text { voyageurs] }\end{array}$ & Ballad Opera 1, 4 \\
\hline Voyer 1928 & Intendant Bigot, L. & Opera 1,4 \\
\hline Willan 1928 & $\begin{array}{l}\text { Ordre de Bon Temps, L'; The Order of } \\
\text { Good Cheer }\end{array}$ & Ballad Opera 1, 4, 5 \\
\hline Wallace 1929 & Bound for the Rio Grande & Ballad Opera 4 \\
\hline Willan 1929a & Indian Christmas [Nativity] Play, An & Ballad Opera 1, 4, 6 \\
\hline Willan 1929b & Prince Charlie and Flora & Ballad Opera 1, 4 \\
\hline $\begin{array}{l}\text { Willan, Gibbon } \\
1929\end{array}$ & Christmas with Herrick & Ballad Opera 6 \\
\hline Létourneau 1930 & Coup de Soleil & Operetta 1, 4 \\
\hline
\end{tabular}


LIST 3: (cont'd)

\begin{tabular}{|c|c|c|}
\hline $\begin{array}{l}\text { Preliminary } \\
\text { Ingraham } \\
\text { Number }\end{array}$ & Title & Genre \\
\hline $\begin{array}{l}\text { Morin-Labrecque } \\
1930\end{array}$ & Francine & Comic Opera 1 \\
\hline O'Brien 1930a & Noce canadienne-française, Une & Ballad Opera 1, 4 \\
\hline O'Brien 1930b & Visions canadiennes: À Saint Malo & Ballad Opera 1 \\
\hline Spencer 1930 & Cavaliers, The & Operetta 1, 4 \\
\hline Willan 1930 & Ayreshire Ploughman, The & Ballad Opera 1 \\
\hline MacMillan 1931 & Prince Charming & Ballad Opera 1, 4 \\
\hline Létourneau 1933 & Mam'zell Bebe & Operetta 1, 4 \\
\hline O'Brien 1933 & Philippino & Comic Opera 1 [Operetta 4] \\
\hline Voyer 1935 & Mademoiselle de Lanaudière & Comic Opera 6 \\
\hline Lapierre 1942 & Père des Amours, Le & Comic Opera 1 [Opéra Comique 4] \\
\hline Willan 1942 & $\begin{array}{l}\text { Transit Through Fire: An Odyssey of } \\
1942\end{array}$ & Radio Opera 1, 4 \\
\hline Faith 1943 & Gandy Dance, The & Operetta 4 \\
\hline Leonard 1943 & Maids of Hamelin & Operetta 4 \\
\hline Willan 1943 & Brébeuf & Pageant 4 \\
\hline Higgin 1945 & Rumplestilskin & Operetta 6 \\
\hline Higgin 1946 & Queen of Romance, The & Operetta 4 \\
\hline $\begin{array}{l}\text { Willan 1946, rev. } \\
1965\end{array}$ & Deirdre of the Sorrows & Radio Opera 1 [Opera 2, 4, 5] \\
\hline Lapierre 1947 & Vagabond de la gloire, Le & Comic Opera 1 [Opéra Comique 4] \\
\hline George 1948 & Evangeline & Opera 2, 3, 4 \\
\hline Poynter 1950 & Triumph of Our Lord, The & Opera 4 \\
\hline Harris 1952 & Portrait of a City & Pageant 4 \\
\hline Leonard 1952 & Girl of Bandolier, The & Operetta 4 \\
\hline Pentland 1952 & Lake, The & Chamber Opera 1, 2, 4 [Opera 5] \\
\hline Jones 1953 & Broken Ring, The & Ballad Opera 1 [Opera 4] \\
\hline
\end{tabular}


LIST 3: (cont'd)

\begin{tabular}{|c|c|c|}
\hline $\begin{array}{l}\text { Preliminary } \\
\text { Ingraham } \\
\text { Number }\end{array}$ & Title & Genre \\
\hline Somers 1953 & Fool, The; Le Fou & Chamber Opera 1, 2 [Opera 4, 5] \\
\hline Blackburn 1954 & $\begin{array}{l}\text { Mesure de silence, Une; A Silent } \\
\text { Measure }\end{array}$ & $\begin{array}{l}\text { Chamber Opera } 1 \text { [Comic Opera 2, 3, } \\
4 ; \text { Opera 5] }\end{array}$ \\
\hline Betts 1955 & Riders to the Sea & Chamber Opera 2 [Opera 4, 5] \\
\hline Somers 1955 & Homeless Ones, The & Operetta 4 \\
\hline Coulthard 1956 & Return of the Native, The & Opera $1,2,3,4$ \\
\hline Beckwith 1958 & Night Blooming Cereus & $\begin{array}{l}\text { Chamber Opera } 1 \text { [Comic Opera 2, 3; } \\
\text { Opera 4, 5] }\end{array}$ \\
\hline Poynter 1959 & Birth of Our Lord, The & Opera 1, 4 \\
\hline Betts 1960 & Woodcarver's Wife, The & Chamber Opera 1 \\
\hline Blackburn 1960 & Pirouette & $\begin{array}{l}\text { Divertissement Chanté } 3 \text { [Chamber } \\
\text { Opera 4] }\end{array}$ \\
\hline George 1960 & Way Out & Opera 4 \\
\hline Sirulnikoff 1960 & This Evening & Opera $1,2,5$ \\
\hline Eggleston 1961 & Woodcarver's Wife, The & Opera 4 \\
\hline Symonds 1961 & Opera for Six Voices & Radio Opera 1, 4 \\
\hline Vallerand 1961 & Magicien, Le & Chamber Opera 1, 4 [Opera 2, 5] \\
\hline Kunz 1962a & Damask Drum, The & Chamber Opera 3, 4, 6 \\
\hline Kunz 1962b & Three Figures in a Crystal Ball & Chamber Opera 6 \\
\hline McPeek 1963 & Bargain, The & $\begin{array}{l}\text { Chamber Opera } 1 \text { [Opera Buffa 2; TV } \\
\text { Opera 4; Opera 5] }\end{array}$ \\
\hline Pannell 1963 & Aria da Capo & Chamber Opera 1, 4 [Opera 2, 5] \\
\hline $\begin{array}{l}\text { Quesnel / Ridout } \\
1963\end{array}$ & Colas et Colinette (reconstruction) & $\begin{array}{l}\text { Comédie en prose mélée } 1 \text { [Vaudeville } \\
\text { Comedy 2] }\end{array}$ \\
\hline Charpentier 1964 & Bourgeois gentilhomme, Le & Comedy-Ballet 2, 3 \\
\hline Detweiler 1964 & Beware of the Wolf & Children's Opera 1 \\
\hline Barnes 1965 & Byron, the Wonderful Bandit & Opera 5 \\
\hline Charpentier 1965 & Klondyke & $\begin{array}{l}\text { Drama Set to Music } 2 \text { [Action } \\
\text { Dramatique 3] }\end{array}$ \\
\hline Jones 1965 & Sam Slick & Chamber Opera 1 [Opera 2, 3, 4, 5] \\
\hline
\end{tabular}


LIST 3: (cont'd)

\begin{tabular}{|c|c|c|}
\hline $\begin{array}{l}\text { Preliminary } \\
\text { Ingraham } \\
\text { Number }\end{array}$ & Title & Genre \\
\hline Kunz 1965a & Let's Make a Carol & Play With Music 3, 4 \\
\hline Kunz 1965b & Watchful Gods, The & Operetta 2, 3, 4 [Opera 5] \\
\hline Polgar 1965 & European Lover, A & $\begin{array}{l}\text { Musical Satire Disguised as an Opera } \\
1,2\end{array}$ \\
\hline Schafer 1965 & Toi; Loving & $\begin{array}{l}\text { Audio-Visual Poem [Musical Poem 1; } \\
\text { Opera 2, 5; TV Opera 4] }\end{array}$ \\
\hline Bissell 1966 & His Majesty's Pie & Operetta 1, 2, 3, 4 [Opera 5] \\
\hline Komorous 1966 & Lady Blancarosa & Opera 4 \\
\hline McIntyre $1966 a$ & Death of a Hired Man, The & Chamber Opera 1, 2 [Opera 4] \\
\hline Mclntyre $1966 b$ & This Is Not True & Comic Opera 1, 2 [Opera 5] \\
\hline Adaskin 1967 & Grant, Warden of the Plains & Chamber Opera 1, 2, 3 [Opera 4, 5] \\
\hline Jones 1967 & Scottish Landing & Nova Scotia Folk Opera 1 \\
\hline Major 1967 & Loyalists, The & Opera 4 \\
\hline Pannell 1967 & Luck of Ginger Coffey, The & Opera $1,4,5$ \\
\hline Somers 1967 & Louis Riel & Opera 1, 2, 4, 5 [Music Drama 3] \\
\hline Turner 1967 & Brideship, The & Lyric Drama 1 [Opera 4, 5] \\
\hline Behrens 1968 & Lay of Trym, The & Music Drama 1, 2, 3 \\
\hline Charpentier 1968 & English Lesson, An; or, Clara-Teacher & $\begin{array}{l}\text { Chamber Opera } 1 \text { [Drama in Music 2, } \\
\text { 3; Opera 5] }\end{array}$ \\
\hline Davies 1968 & Let Us Pay Tribute to Lord Gordon & Opera 4 \\
\hline Polgar 1968 & Troublemaker, The & Comic Opera 1, 2 [Opera 5] \\
\hline Burritt 1969 & Once Again . . . Pop! & Pantomime 4 \\
\hline Charpentier 1969 & Orphée I & Opera 4, 5 \\
\hline Goldberg 1969 & Galatea elettronica & Chamber Opera 4 \\
\hline $\begin{array}{l}\text { Benoist n.d. } \\
\text { Rencontre }\end{array}$ & Rencontre dans l'escalier, La & Opera 4 \\
\hline Benoist n.d. Saint & Saint-François d'Assise & Opera 4 \\
\hline $\begin{array}{l}\text { Benoist n.d. } \\
\text { Secret }\end{array}$ & Secret des Amati, Le & Operetta 4 \\
\hline
\end{tabular}


LIST 3: (cont'd)

\begin{tabular}{|c|c|c|}
\hline $\begin{array}{l}\text { Preliminary } \\
\text { Ingraham } \\
\text { Number }\end{array}$ & Title & Genre \\
\hline Cabena 1970 & Selfish Giant, The & Opera 3, 4 \\
\hline Goldberg 1970 & Concrete Rose, The & Rock Opera 4 \\
\hline Kenins 1970 & Lagalai-Legend of the Stone & Chamber Opera 3 \\
\hline Polgar 1970 & Strange Night, A & Opera 2 \\
\hline Burritt 1971 & Electric Chair & Pantomime 4 \\
\hline Mann 1971 & Donkey's Tale, The & Chamber Opera 2 \\
\hline Schafer 1971 & In Search of Zoroaster & Opera 6 \\
\hline Symonds 1971 & $\begin{array}{l}\text { Charnisay Versus La Tour or, The Spirit } \\
\text { of Fundy }\end{array}$ & $\begin{array}{l}\text { Chamber Opera } 1 \text { [Operatic melodrama } \\
\text { 2; Opera 4] }\end{array}$ \\
\hline Buczynski 1972 & $\begin{array}{l}\text { From The [Buczynski] Book of the } \\
\text { Living }\end{array}$ & Chamber Opera 1, 2, 3 [Opera 4, 5] \\
\hline $\begin{array}{l}\text { Charpentier } \\
1972 \mathrm{a}\end{array}$ & Orphée II & Liturgy 1 [Opera (rev. from 1969) 5] \\
\hline $\begin{array}{l}\text { Charpentier } \\
1972 \mathrm{~b}\end{array}$ & $\begin{array}{l}\text { Tea Symphony, The; or, The Perils of } \\
\text { Clara }\end{array}$ & Kitsch Opera 2, 3 [Opera 5] \\
\hline Dolin 1972 & Drakkar & Entertainment 2 [Opera 5] \\
\hline Ford 1972 & Hypnos & Lyric Drama 1, 2, 3 [Opera 5] \\
\hline Schafer 1972 & Patria 2: Requiems for the Party Girl & Opera Happening 6 \\
\hline Wilson $1972 \mathrm{a}$ & Héloise and Abélard & Opera $1,2,4,5$ \\
\hline Wilson 1972b & Selfish Giant, The & Children's Opera 1, 4, 5 [Opera 2] \\
\hline Wilson 1972c & Summoning of Everyman, The & Church Opera 1 [Opera 2, 4, 5] \\
\hline Archer 1973 & Sganarelle & $\begin{array}{l}\text { Comic Opera 1, } 4 \text { [Chamber Opera 2, } \\
\text { 3; Opera 4, 5] }\end{array}$ \\
\hline Pannell 1973 & Exiles & Opera for the Theatre 1 [Opera 4] \\
\hline Polgar 1973 & Glove, The & Comic Opera 1, 2 [Opera 4, 5] \\
\hline Rae 1973 & Like Gods Among Them & Dance Drama 6 \\
\hline Rea 1973 & Prisoner's Play, The & Chamber Opera 1, 2 [Opera 4, 5] \\
\hline Benoist 1974 & Légende du vent, La & Lyric Drama 4 \\
\hline Crawley 1974 & Slaughter of the Innocents, The & Opera 2, 3 \\
\hline
\end{tabular}


LIST 3: (cont'd)

\begin{tabular}{|c|c|c|}
\hline $\begin{array}{l}\text { Preliminary } \\
\text { Ingraham } \\
\text { Number }\end{array}$ & Title & Genre \\
\hline Davies 1974 & Beowulf & Rock Opera 4 \\
\hline Schafer 1974 & Characteristics Man, The & Work For Theatre 6 [Opera 5] \\
\hline Anhalt 1975 & Tourangelle, La & Opera 4, 5 \\
\hline Doolittle 1975 & Charlie the Chicken & Operatic Entertainment 2, 3 [Opera 4] \\
\hline George 1975 & King for Corsica, $A$ & Opera $1,2,3,4$ \\
\hline Pannell 1975a & Go & Opera 4 \\
\hline Pannell 1975b & Midway & Opera 4 \\
\hline Rogers 1975 & So Hard to Be So Hard & Radio Folk Opera 4 \\
\hline Wilson 1975 & Kamouraska & Opera $1,2,4,5$ \\
\hline Charpentier 1976 & $\begin{array}{l}\text { Clara et les philosophes; Clara and the } \\
\text { Philosophers }\end{array}$ & Cocktail Opera 1 \\
\hline Klein 1976 & Orpheus & Lyrical Essay 3 \\
\hline Pannell 1976 & Push & Opera 4 \\
\hline Ridout 1976 & Lost Child, The & Opera 1, 2, 5 [TV Opera 4] \\
\hline Schafer 1976 & Apocalypsis, part 2: Credo & Opera 6 \\
\hline Benoist 1977 & Onadéga ou la tragédie de lac des bois & Opera 4 \\
\hline Bissell 1977 & Musical Play, A & Operetta 4 \\
\hline Healey 1977 & Seabird Island & Opera 1, 2, 3, 4, 5 \\
\hline Maclntyre 1977 & Humulus the Mute & Opera 2 \\
\hline Pannell 1977a & Aberfan & $\begin{array}{l}\text { Chamber Opera } 1 \text { [TV Opera 4; Opera } \\
\text { 5] }\end{array}$ \\
\hline Pannell 1977b & Circe & Masque 1,4 \\
\hline Pannell 1977c & $N-E-W-S$ & Opera 4 \\
\hline Riley 1977 & Mandragola & [Musical setting of Libretto] 4 \\
\hline Schafer 1977 & Apocalypsis, part 1: John's Vision & Opera 6 \\
\hline Somers 1977 & Death of Enkidu & Opera 4, 5 \\
\hline
\end{tabular}


LIST 3: (cont'd)

\begin{tabular}{|c|c|c|}
\hline $\begin{array}{l}\text { Preliminary } \\
\text { Ingraham } \\
\text { Number }\end{array}$ & Title & Genre \\
\hline Symonds 1977 & Lady in the Night & Opera 4, 5 \\
\hline Wilson 1977 & Psycho Red & $\begin{array}{l}\text { Dramatic "Mindscape" Within Music 1, } 2 \\
\text { [Opera 4, 5] }\end{array}$ \\
\hline Beckwith 1978 & Shivaree, The & Chamber Opera 1, 2 [Opera 3, 4, 5] \\
\hline Buczynski 1978 & Naked at the Opera & Opera 1, 2, 3 \\
\hline Freedman 1978 & Silents! & Comic Opera 4 \\
\hline Keane 1978 & $\begin{array}{l}\text { Devil's Constructs, The; or, The Eighth } \\
\text { Day }\end{array}$ & $\begin{array}{l}\text { Anatomical Electronical Musical (or, a } \\
\text { Sci-fi Opera) } 1 \text { [Chamber Opera 2, 3; } \\
\text { Opera 4] }\end{array}$ \\
\hline Levin 1978 & Rebel and Empire & Opera $1,2,4$ \\
\hline $\begin{array}{l}\text { Mcley 1978, rev } \\
1996\end{array}$ & Salmon Dance & Dance, Music And Poetry Drama 6 \\
\hline $\begin{array}{l}\text { Pannell 1978, rev. } \\
1984\end{array}$ & $\begin{array}{l}\text { Souvenirs [As Long As a Child } \\
\text { Remembers] }\end{array}$ & Chamber Opera 4 \\
\hline Symonds 1978 & Episode at Big Quill & Music Drama 1 [Radio Theatre 4] \\
\hline Charpentier 1979 & Clarabelle-Clarimage & Opera 4 \\
\hline Farrell 1979 & Birthday of the Infanta, The & Opera 4 \\
\hline Freedman 1979 & Abracadabra & $\begin{array}{l}\text { Comic/Jazz Opera (Jazz Chamber } \\
\text { Opera) } 1 \text { [Opera 2, 3, 4] }\end{array}$ \\
\hline Hambraeus 1979 & Sagan (The Legend) & Radio Opera 1, 3 [Opera 2] \\
\hline Kunz 1979 & Ceyx and Alcyone & Chamber Opera 1, 2, 3 [Opera 4] \\
\hline Pannell 1979 & Refugees & Vaudeville Opera 1, 4 \\
\hline Schafer 1979a & Beauty and the Beast & Opera 2 \\
\hline Schafer 1979b & Jonah & Musical-Dramatic Work 2, 6 \\
\hline Vivier 1979 & Kopernikus & $\begin{array}{l}\text { Chamber Opera } 1 \text { [Ritual Opera of } \\
\text { Death 2; Opera 4] }\end{array}$ \\
\hline Bissell 1980 & Miraculous Turnip, The & Opera 1, 2, 3 [Children's Opera 4] \\
\hline Crawley 1980 & Barnardo Boy & Opera 1, 2, 3, 4 \\
\hline Doolittle 1980 & Second Shepherds' Play, The & Opera 2 \\
\hline Pannell 1980 & Harvest & Chamber Opera 1 [TV Opera 4] \\
\hline
\end{tabular}


LIST 3: (cont'd)

\begin{tabular}{|c|c|c|}
\hline $\begin{array}{l}\text { Preliminary } \\
\text { Ingraham } \\
\text { Number }\end{array}$ & Title & Genre \\
\hline Raum 1980 & Final Bid, The & Opera 2, 4 \\
\hline Wuensch 1980 & Amadeus Revisited & Opera Fragment 2 \\
\hline Lamarche 1981 & Mira & Opera 4 \\
\hline Maclntyre 1981 & Refrains & Opera 3 \\
\hline Schafer 1981 & $\begin{array}{l}\text { Patria: The Princess of Stars: The } \\
\text { Prologue }\end{array}$ & $\begin{array}{l}\text { Ritual for Performance at Dawn on a } \\
\text { Fall Morning on a Lake 2, } 6\end{array}$ \\
\hline Holman 1982 & Doctor Canon's Cure & Opera 2, 3 [Children's Opera 4] \\
\hline Morgan 1982 & Nobleman's Wedding, The & Folk Opera 6 \\
\hline Turner 1982 & Vile Shadows & Opera 2 \\
\hline Adaskin 1983 & Traveling Musicians, The & Opera 4 \\
\hline Archer 1983 & Meal, The & Opera 2, 3, 4 \\
\hline Burritt 1983 & Altar of the Sun & Musical 2 [Mixed Media 4] \\
\hline Deschênes 1983 & OPéRAaaAH! & Opera 4 \\
\hline Frenette 1983 & Étrivières, Les & Opera 2, 4 \\
\hline Hannah 1983 & Madamoiselle Fifi & Chamber Opera 2, 3 \\
\hline Henderson 1983 & Clear Sky \& Thunder & Inuit Music Drama 2 \\
\hline Keane 1983 & Carmina Tenebrarum & Opera 4 \\
\hline Raum 1983 & Garden of Alice, The & Opera 2, 4 \\
\hline Schafer 1983 & Patria 6: RA & $\begin{array}{l}\text { Multi-Sensory Experience, Series of } \\
\text { Rituals } 6\end{array}$ \\
\hline Doolittle 1984 & Silver City & Opera $2,3,4$ \\
\hline $\begin{array}{l}\text { Jaeger, Lake, } \\
\text { Montgomery } \\
1984\end{array}$ & Nightbloom & Opera 4 \\
\hline $\begin{array}{l}\text { Saint-Marcoux } \\
1984\end{array}$ & Transit & Opera 4 \\
\hline Shepherd 1984 & $\begin{array}{l}\text { Hood of the Woods Meets the Dark } \\
\text { Lady of the Sonata, The }\end{array}$ & Pocket Opera 2 \\
\hline Charpentier 1985 & Claracello ou Répertoire & Opera 4 \\
\hline Levin 1985 & Ghost Dance & Opera 4 \\
\hline
\end{tabular}


LIST 3: (cont'd)

\begin{tabular}{|c|c|c|}
\hline $\begin{array}{l}\text { Preliminary } \\
\text { Ingraham } \\
\text { Number }\end{array}$ & Title & Genre \\
\hline Anhalt 1986 & Winthrop & Musical Pageant 3 [Opera 4] \\
\hline Bottenberg 1986 & Inook & Opera 2, 3 \\
\hline Gagné 1986 & Menaud (Master Logdriver) & $\begin{array}{l}\text { Opera } 2 \text { [Poème Dramatique: Opera 3; } \\
\text { Opera 4] }\end{array}$ \\
\hline Hambraeus 1986 & Ouï-dire, $L^{\prime}$ & Prologue and Scene 2, 3 [Opera 4] \\
\hline Raum 1986 & Thunder in A Concave Vacuum & Multi-Media Theatre Work 2 \\
\hline Arsenault 1987 & Aristocratic Lover, The & Dramatic Musical 2 \\
\hline George 1987 & Revolt in Upper Canada & Opera 4 \\
\hline Pelletier 1987 & Studio variazioni sull'a & Dramatic Fantasy 2 \\
\hline Provost 1987 & Phaedra & Opera 4 \\
\hline Rae 1987 & C.3.3. & Music Theatre 3 [Chamber Opera 6] \\
\hline Schafer 1987a & $\begin{array}{l}\text { Patria 1: Wolfman (formerly, The } \\
\text { Characteristics Man) }\end{array}$ & $\begin{array}{l}\text { Opera/Greek Drama/Comedy of Errors/ } \\
\text { Thriller/Study of Alienation [Work for the } \\
\text { Theatre } 1,2 \text { ] }\end{array}$ \\
\hline Schafer 1987b & Patria 3: The Greatest Show & $\begin{array}{l}\text { Society of } 64 \text { Members ( } 8 \text { Clans) Who } \\
\text { Perform the Work Annually } 6\end{array}$ \\
\hline Sullivan 1987 & Tomorrow and Tomorrow & Opera 2, 4 \\
\hline Beckwith 1988 & Crazy to Kill & Detective Opera 2, 3 [Opera 4] \\
\hline Brégent 1988 & Réalitillusion & Chamber Opera 2, 3 [Opera 4] \\
\hline Désilets 1988 & Zoé & $\begin{array}{l}\text { Chamber Opera } 2 \text { [Fable Lyrique 3; } \\
\text { Opera 4] }\end{array}$ \\
\hline Doolittle 1988 & Boiler Room Suite & Opera 2, 3, 4 \\
\hline Hodkinson 1988 & Saint Carmen of the Main & Opera 4 \\
\hline Komorous 1988 & No No Miya & Opera 4 \\
\hline Schafer 1988a & $\begin{array}{l}\text { Patria 4: The Black Theatre of Hermes } \\
\text { Trismegistos }\end{array}$ & Theatre \\
\hline Schafer 1988b & $\begin{array}{l}\text { Patria Epilogue: And Wolf Shall Inherit } \\
\text { the Moon }\end{array}$ & $\begin{array}{l}\text { Society Of } 64 \text { Members ( } 8 \text { Clans) Who } \\
\text { Perform The Work Annually } 6\end{array}$ \\
\hline Somers 1988 & Serinette & Chamber Opera 2 [Opera 4] \\
\hline Sullivan 1988 & Dream Play & Opera 2, 4 \\
\hline
\end{tabular}


LIST 3: (cont'd)

\begin{tabular}{|c|c|c|}
\hline $\begin{array}{l}\text { Preliminary } \\
\text { Ingraham } \\
\text { Number }\end{array}$ & Title & Genre \\
\hline Crawley 1989 & Pied Piper, The & Opera 4 \\
\hline Gougeon 1989 & Expensive Embarrassment, An & Chamber Opera 2 [Opera 3, 4] \\
\hline Koprowski 1989 & Dulcitius: Demise of Innocence & Opera 4 \\
\hline MacDonald 1989 & Unbelievable Glory of Mr. Sharp, The & Opera 4 \\
\hline Miller 1989 & Around Alline & Opera 4 \\
\hline Patenaude 1989 & Pour ces quelques arpents de neige & Opera 4 \\
\hline Shepherd 1989 & Serrure, La & Operatic Morceau 2 \\
\hline Désilets 1990 & Zaria & Opera 2 [Overture et Scènes 3] \\
\hline Evangelista 1990 & Alice and Friends & Monodrama 2 \\
\hline Gagnon 1990 & Nelligan & Opera 4 \\
\hline Lake 1990 & Tis Pity & Opera 4 \\
\hline Pannell 1990 & Animals of Limbo, The & Christmas Pageant 4 \\
\hline Raum 1990 & $\begin{array}{l}\text { Eos, The Dream of Nicholas Flood } \\
\text { Gavin }\end{array}$ & Opera 2, 4 \\
\hline Oliver 1991 & Guacamayo's Old Song and Dance & Opera 4 \\
\hline Schafer 1991 & Patria 5: The Crown of Ariadne & $\begin{array}{l}\text { Retelling Of The Tale Of Theseus And } \\
\text { The Minotaur } 6\end{array}$ \\
\hline Somers 1991a & Mario and the Magician & Opera 2, 4 \\
\hline Somers 1991b & Midwinter Night's Dream, A & Opera 2 [Children's Opera 4] \\
\hline Sullivan 1991 & Florence & Opera 4 \\
\hline Thibault 1991 & Ne blâmez jamais les bédouins & Chamber Opera 2 [Opera 4] \\
\hline Evangelista 1992 & $\begin{array}{l}\text { Exercices de conversation et de diction } \\
\text { françaises pour étudiants américains }\end{array}$ & Chamber Opera 3 \\
\hline Gotham 1992 & Nigredo Hotel & Chamber Opera 2, 3 [Opera 4] \\
\hline Hétu 1992 & Prix, Le & Opera $2,3,4$ \\
\hline Provost 1992 & Adorable verrotière, $L^{\prime}$ & Monodrama 2 \\
\hline Beckwith 1993 & Taptoo! & Opera 3, 4 \\
\hline
\end{tabular}


LIST 3: (cont'd)

\begin{tabular}{|c|c|c|}
\hline $\begin{array}{l}\text { Preliminary } \\
\text { Ingraham } \\
\text { Number }\end{array}$ & Title & Genre \\
\hline Burry 1993 & Unto the Earth: Vignettes of a War & Opera 6 \\
\hline Kulesha 1993 & Red Emma & Opera 3, 4 \\
\hline Mather 1993 & Princesse blanche, La & Chamber Opera 2 [Opera 4] \\
\hline Rickard 1993 & Fletcher's Challenge & Opera/Pageant 3 \\
\hline Peters 1993 & Nosferatu & Opera 4 \\
\hline Gagné 1994 & Évangéline et Gabriel & Opera 2, 3, 4 \\
\hline Maclntyre 1994 & Architect, The & Opera 3 \\
\hline Schafer 1994 & Patria 9: The Enchanted Forest & Interactive Story In A Forest 6 \\
\hline Underhill 1994 & Star Catalogues, The & Opera 6 \\
\hline Patenaude 1995 & Chevalier de Lorimier & Opera 4 \\
\hline Anhalt 1996 & Traces (Tikkum) & Pluri-Drama 3 [Opera 4] \\
\hline Burry 1996 & Shanawdithit & Opera 6 \\
\hline Crawley 1996 & Angel Square & Opera 3, 4 \\
\hline Luedeke 1996 & $\begin{array}{l}\text { Oscar Excerpts: The Picture of Dorian } \\
\text { Gray }\end{array}$ & Opera 3 \\
\hline Provost 1996 & Vampire et la nymphomane, Le & Opera 4 \\
\hline Rolfe 1996 & Beatrice Chancey & Opera 4 \\
\hline Badian 1997 & Scenic Mosaics & Pantomime 3 \\
\hline Désilets 1997 & Zaâ le loup & Theatre Piece 2 [Opéra Poétique 3] \\
\hline Piché 1997 & Yo soy la Desintegracion & Opera 3 \\
\hline Schafer 1997 & $\begin{array}{l}\text { Patria 10: The Spirit Garden Part I: } \\
\text { Spring }\end{array}$ & $\begin{array}{l}\text { A Ritual In Which A Garden Is Prepared } \\
\text { And Planted } 6\end{array}$ \\
\hline Butterfield 1998 & Zurich 1916 & Opera 6 \\
\hline Hatch 1998 & Asks Alice & Micro-Opera 3 \\
\hline Sharman 1998 & Elsewhereless & Opera 4 \\
\hline Wiens 1998 & Down Here on Earth & Opera 6 \\
\hline
\end{tabular}


LIST 3: (cont'd)

\begin{tabular}{|c|c|c|}
\hline $\begin{array}{l}\text { Preliminary } \\
\text { Ingraham } \\
\text { Number }\end{array}$ & Title & Genre \\
\hline Hannan 1999 & Gang, The & Opera 4 \\
\hline Kulesha 1999 & Last Duel, The & Opera 3, 4 \\
\hline Parker 1999 & Visitor, The & Chamber Opera 3 \\
\hline Peters 1999 & Golden Ass, The & Opera 4 \\
\hline Anhalt 2000 & Millenial Mall (Lady Diotima's Walk) & $\begin{array}{l}\text { Voice-Drama for the Imagination } 3 \\
\text { [Opera 4] }\end{array}$ \\
\hline Applebaum 2000 & Erehwon & Opera 4 \\
\hline Chan 2000 & Iron Road & Opera 4 \\
\hline Greer 2000 & Star-Child, The & Children's Opera 4 \\
\hline McIntyre 2000 & Thirteen Hands & Opera 3 \\
\hline Richardson 2000 & Illusion, The & Opera 6 \\
\hline Uyeda 2000 & Game Misconduct & Opera 4 \\
\hline Bell 2001 & Turtle Wakes & Opera 4 \\
\hline Buck 2001 & Bones & Dance Opera 6 \\
\hline Burry 2001 & Brothers Grimm, The & Children's Opera 4, 6 \\
\hline Donison 2001 & Eyes on the Mountain & Opera 4 \\
\hline Duncan 2001 & Fall of the House of Usher & Opera \\
\hline Evangelista 2001 & Manuscrit trouvé à Saragosse & Opera 4 \\
\hline Gagné 2001 & Verdi, Les & Opera 3, 4 \\
\hline Louie 2001 & Toothpaste & Opera Buffa 4 \\
\hline Schafer 2001a & $\begin{array}{l}\text { Patria 8: The Palace of Cinnabar } \\
\text { Phoenix }\end{array}$ & $\begin{array}{l}\text { Interactive Story In The Forest At The } \\
\text { Edge Of A Lake } 6\end{array}$ \\
\hline Schafer 2001b & $\begin{array}{l}\text { Patria 10: The Spirit Garden Part II: } \\
\text { Autumn }\end{array}$ & Harvest And Banquet Rituals 6 \\
\hline Daniel 2002 & Lisa & Horror Opera 3 [Mini-Opera 6] \\
\hline Hannan 2002 & 120 Songs for the Marquis de Sade & Opera 4 \\
\hline Hui 2002 & Cellar Door, The & Opera 6 \\
\hline
\end{tabular}


LIST 3: (cont'd)

\begin{tabular}{|c|c|c|}
\hline $\begin{array}{l}\text { Preliminary } \\
\text { Ingraham } \\
\text { Number }\end{array}$ & Title & Genre \\
\hline Louie 2002 & Scarlet Princess, The & Opera 4 \\
\hline Richardson 2002 & Seven Stories & Opera 6 \\
\hline Ryan 2002 & Laurels, The & Opera 6 \\
\hline Burritt 2003 & Pilgrim [The Dream Healer] & Opera 3 \\
\hline Danielson 2003 & Mariken of Nimmegen & Opera 6 \\
\hline Estacio 2003 & Filumena & Opera 3, 4 \\
\hline Jackson 2003 & Québécité & Opera 4 \\
\hline Palmer 2003 & Over the Japanese Sea & Opera 6 \\
\hline Ryan 2003 & Ghost in the Turrett, The & Children's Opera 4 \\
\hline Smith 2003 & Facing South & Opera 6 \\
\hline Burry 2004 & Hobbit, The & Children's Opera 4, 6 \\
\hline Chan 2004 & Ice Time & Opera 6 \\
\hline Daniel 2004 & Via Cruxus & Opera 6 \\
\hline Duncan 2004 & Belle et la Bête, La & Chamber Opera 3 [Opera 4] \\
\hline Ferguson 2004 & Two Graces, The & Opera 6 \\
\hline Hatzis 2004 & Constantinople & $\begin{array}{l}\text { Opera } 4 \text { [Multimedia Music Theatre } \\
\text { Work 6] }\end{array}$ \\
\hline Peters 2004 & Inanna's Journey & Opera 6 \\
\hline Richardson 2004 & Mother Everest & Opera 6 \\
\hline Rolfe 2004a & Charlotte & Opera 6 \\
\hline Rolfe 2004b & Orpheus and Eurydice & Masque 3 \\
\hline Rolfe 2004c & Rosa & Chamber Opera 3 \\
\hline Luengen 2005 & Naomi's Road & Children's Opera 4 \\
\hline Sokolovic 2005 & Midnight Court Opera, The & Opera 6 \\
\hline Staniland 2005 & Ashlike on the Cradle of the Wind & Chamber Opera 6 \\
\hline
\end{tabular}


LIST 3: (cont'd)

\begin{tabular}{|c|c|c|}
\hline $\begin{array}{l}\text { Preliminary } \\
\text { Ingraham } \\
\text { Number }\end{array}$ & Title & Genre \\
\hline Burry 2006a & Isis and the Seven Scorpions & Opera 6 \\
\hline Burry 2006b & Vinland Traveler, The & Opera 6 \\
\hline Current 2006a & Airline Icarus & Chamber Opera 6 \\
\hline Current 2006b & Inventory & Chamber Opera 6 \\
\hline Donison 2006 & Island & Opera 4 \\
\hline Estacio 2006 & Frobisher & Opera 6 \\
\hline Louie 2006 & $\begin{array}{l}\text { Burnt Toast: } 8 \text { mini comic operas about } \\
\text { love }\end{array}$ & TV Opera 4 \\
\hline Rolfe $2006 a$ & Elijah's Kite & Opera 6 \\
\hline Rolfe $2006 b$ & Swoon & Opera 3 \\
\hline Truax 2006 & Powers of Two: The Artist & Opera 6 \\
\hline Daniel 2007 & Shadow, The & Opera 6 \\
\hline Gilliland 2007 & Hannaraptor & Opera 6 \\
\hline Jackson 2007 & Trudeau: Long March/Shining Path & Opera 6 \\
\hline Ogborn 2007 & Opera on the Rocks & Opera 6 \\
\hline Palmer 2007 & Shelter & Opera 3 \\
\hline Rolfe 2007 & Aenaes and Dido & Opera 6 \\
\hline $\begin{array}{l}\text { Charpentier n.d. } \\
\text { Ballade }\end{array}$ & Ballade du fils de l'homme & Opera 4 \\
\hline $\begin{array}{l}\text { Charpentier n.d. } \\
\text { Clara Bridge }\end{array}$ & Clara on the Bridge & Opera 4 \\
\hline $\begin{array}{l}\text { Charpentier n.d. } \\
\text { Clara Committee }\end{array}$ & Clara-Committee & Opera 4 \\
\hline $\begin{array}{l}\text { Charpentier n.d. } \\
\text { Clara masque }\end{array}$ & Clara, un masque & Opera 4 \\
\hline $\begin{array}{l}\text { Charpentier n.d. } \\
\text { Clara Nuit }\end{array}$ & Clara, la nuit & Opera 4 \\
\hline $\begin{array}{l}\text { Schafer n.d. } \\
\text { Asterion }\end{array}$ & Patria 7: Asterion & Pataphysical Hierophony 6 \\
\hline
\end{tabular}




\begin{tabular}{||r|l|l||}
\hline \multicolumn{1}{|c|}{$\begin{array}{c}\text { Source } \\
\text { Identification }\end{array}$} & \multicolumn{1}{|c||}{ Title } & \multicolumn{1}{|c||}{ Genre } \\
\hline & Key to List 3 citations \\
\hline 1 & $\begin{array}{l}\text { Cooper, Dorith. 1984. Opera in Montreal and Toronto: A Study of Performance } \\
\text { Traditions and Repertoire, 1783-1980. PhD diss, University of Toronto. }\end{array}$ \\
\hline 2 & $\begin{array}{l}\text { Canadian Music Centre. 1999. Canadian Operas: An Annotated Catalogue of } \\
\text { Operas and Staged Vocal Works by Canadian Composers in the Canadian Music } \\
\text { Centre. Vancouver: Canadian Music Centre. }\end{array}$ \\
\hline 3 & Canadian Music Centre. 2008. Canadian Music Centre. <http://musiccentre.ca>. \\
\hline 4 & $\begin{array}{l}\text { Historica Foundation. 2008. Encyclopedia of Music in Canada. } \\
<\text { http://www.thecanadianencyclopedia.com>. }\end{array}$ \\
\hline 5 & $\begin{array}{l}\text { Proctor, George. 1980. Canadian Music of the Twentieth Century. Toronto: } \\
\text { University of Toronto Press. }\end{array}$ \\
\hline 6 & Other: information from publisher or composer. \\
\hline &
\end{tabular}




\section{REFERENCE LIST}

Borroff, Edith. 1984. "Origin of Species: Conflicting Views of American Musical Theatre History." American Music 2, no. 4 (Winter): 101-112.

Bourdieu, Pierre. 1993. The Field of Cultural Production: Essays on Art and Literature. Edited and introduced by Randal Johnson. New York: Columbia University Press.

Canada Council. 1958. The Canada Council First Annual Report to March 31, 1958. Ottawa: The Canada Council.

Canadian Broadcasting Corporation. 2008. Canadian Broadcasting Corporation Archives. <http://www.cbc.ca/radiocommissions/timeline.html>

Canadian Music Centre. 1999. Canadian Operas: An Annotated Catalogue of Operas and Staged Vocal Works by Canadian Composers in the Canadian Music Centre. Vancouver: Canadian Music Centre.

-2008. Canadian Music Centre. <http://www.musiccentre.ca>

Cooper, Dorith. 1984. Opera in Montreal and Toronto: A Study of Performance Traditions and Repertoire, 1783-1980. PhD diss, University of Toronto.

Corse, Sarah. 1997. Nationalism and Literature: The Politics of Culture in Canada and the United States. New York: Cambridge University Press.

Elliott, R. and Smith, G., eds. 2001. Istvan Anhalt: Pathways and Memories. Montreal and Kingston: McGill-Queen's University Press.

Frye, N. 1957. Anatomy of Criticism: Four Essays. Princeton, NJ: Princeton University Press.

Government of Canada. 2008. Library and Archives Canada. <http://www.collectionscanada.gc.ca>.

Historica Foundation. 2007. Encyclopedia of Music in Canada. <http://www. thecanadianencyclopedia.com>.

Hutcheon, L. 1990. As Canadian as ... possible ... under the circumstances! North York: York University Press.

Hutcheon, L., and M. Hutcheon. 1995. "Otherhood Issues: Post-national Operatic Imperatives." Narrative, 3.1 (January): 1-17.

Kallmann, Helmut. 1960. A History of Music in Canada 1534-1914. Toronto: University of Toronto Press.

Keillor, Elaine. 2006. Music in Canada. Capturing Landscape and Diversity. Montreal and Kingston: McGill-Queen's University Press.

Mackey, Eva. 1999. The House of Difference: Cultural Politics and National Identity in Canada. London: Routledge.

Proctor, George. 1980. Canadian Music of the Twentieth Century. Toronto: University of Toronto Press.

Said, Edward. 1993. Culture and Imperialism. New York: Knopf.

Straw, W. 2005. "Pathways of Cultural Movement." In Accounting for Culture: Thinking Through Cultural Citizenship. Edited by S. Jeannotte, M. Gattinger, C. Andrews and W. Straw. Ottawa: University of Ottawa Press, 183-97.

Williams, Bernard. 2008. "Opera, III, 1" Grove Music Online (accessed 24 January 2008), <http://www.grovemusic.com/>. 


\section{ABSTRACT}

The primary aim of this article is to introduce and present a preliminary list of Canadian staged dramatic music composed between 1867 and 2007 as a contribution to a previously under-cultivated field of Canadian cultural activity and research. More than simply an exercise in establishing a repertoire, this article approaches these works as important historical documents that articulate contemporaneous cultural, social, and political values over the past 140 years. Through a discussion of issues raised in determining genre and musical citizenship, the scope, nature, and history of these works lays a foundation for systematic research into this most important Canadian cultural artifact.

To facilitate access to what is a substantial body of information, this preliminary list is offered in three formats: 1) alphabetically by composer's surname; 2) alphabetically by the title of the work; and 3) chronologically by date of completion of the work. Compositions are identified across all formats by a Preliminary Ingraham Number (PIN) comprised of the composer's surname and the date of completion of the work.

\section{RÉSUMÉ}

Le but de cet article est de présenter - à titre de contribution à un champ d'activités culturelles et de recherche trop souvent négligé - une liste préliminaire d’œuvres musicales dramatiques composées pour la scène entre 1867 et 2007. Au-delà du simple exercice de recensement d'un répertoire, cet article considère ces œuvres comme d'importants documents historiques articulant les valeurs contemporaines culturelles, sociales et politiques depuis les 140 dernières années. Suite à l'évaluation des enjeux apparus en déterminant le genre et la citoyenneté musicale des œuvres, leur ampleur, la nature et l'histoire de celles-ci constituent une solide base permettant la recherche systématique au sein de cet important artefact culturel canadien.

Afin de faciliter l'accès à ce corpus substantiel, la liste préliminaire est proposée en trois formats : 1) en ordre alphabétique de noms de compositeurs; 2) en ordre alphabétique des titres de compositions; 3 ) en ordre chronologique d'achèvement de l'œuvre. Dans les trois listes, les compositions comportent un identifiant ( PIN » pour Preliminary Ingraham Number) composé du nom du compositeur et de la date d'achèvement de l’ouvre. 